\title{
Soil-Structure Interaction
}

\author{
Alexander Tyapin
}

Additional information is available at the end of the chapter

http://dx.doi.org/10.5772/48333

\section{Introduction}

First the very definition of the soil-structure interaction (SSI) effects is discussed, because it is somewhat peculiar: every seismic structural response is caused by soil-structure interaction forces, but only in certain situations they talk about soil-structure interaction (SSI) effects. Then a brief history of this research field is given covering the last 70 years. Basic superposition of wave fields is discussed as a common basis for different approaches direct and impedance ones, first of all. Then both approaches are described and applied to a simple 1D SSI problem enabling the exact solution. Special attention is paid to the substitution of the boundary conditions in the direct approach often used in practice. Impedance behavior is discussed separately with principal differentiation of quasi-homogeneous sites and sites with bedrock. Locking and unlocking of layered sites is discussed as one of the main wave effects. Practical tools to deal with SSI are briefly described, namely LUSH, SASSI and CLASSI. Combined asymptotic method (CAM) is presented.

Nowadays SSI models are linear. Nonlinearity of the soil and soil-structure contact is treated in a quasi-linear way. Special approach used in SHAKE code is discussed and illustrated. Some non-mandatory additional assumptions (rigidity of the base mat, horizontal layering of the soil, vertical propagation of seismic waves) often used in SSI, are discussed. Finally, two of the SSI effects are shown on a real world example of the NPP building. The first effect is soil flexibility; the second effect is embedment of the base mat. Recommendations to engineers are summed in conclusions.

\section{Peculiarities of the SSI definition}

Soil-structure interaction (SSI) analysis is a special field of earthquake engineering. It is worth starting with definition. Common sense tells us that every seismic structural response is caused by soil-structure interaction forces impacting structure (by the definition of seismic excitation). However, engineering community used to talk about soil-structure interaction 
only when these interaction forces are able to change the basement motion as compared to the free-field ground motion (i.e. motion recorded on the free surface of the soil without structure). So, historically the conventional definition of SSI is different from simple occurrence of the interaction forces: these forces occur for every structure, but not always they are able to change the soil motion.

This simple fact leads to the important consequences. If a structure can be analyzed as based on rigid foundation with free-field motion at it, then they use to say that "no SSI effects occur" (though structure is in fact moved by the interaction forces, and the same forces impact the foundation). Looking at the variety of the real-world situations, we can conclude that only part of them satisfies the conventional definition of SSI.

The ability of the interaction forces to change the soil motion depends, of course, on two factors: value of the force and flexibility of the soil foundation. The value of the interaction force may be often estimated via the base mat acceleration and inertia of the structure. For given soil site and given free-field seismic excitation the heavier is the structure, the more likely SSI effects occur. Usually most of civil structures resting on hard or medium soils do not show the signs of considerable SSI effects.

From the inertial point of view, the heaviest structures we deal with are hydro-structures (like dams) and nuclear power plant (NPP) main structures - first of all, reactor buildings. So, the development of the SSI field in the earthquake engineering was historically linked to the development of these two fields of industry.

From the soil flexibility point of view, for the given structure and given free-field seismic excitation the softer is the soil, the more likely SSI effects occur. Soil shear module is a product of mass density and square of the shear wave velocity. Mass density of soil in practice varies around 2,0 t/m $\mathrm{m}^{3}$ in a comparatively narrow range, so the main characteristic of the soil stiffness is shear wave velocity $V_{s}$. Usually soil is considered "soft" when $\mathrm{V}_{\mathrm{s}}$ is less than $300 \mathrm{~m} / \mathrm{s}$, and "hard" when $\mathrm{V}_{\mathrm{s}}$ is greater than $800 \mathrm{~m} / \mathrm{s}$. If $\mathrm{V}_{\mathrm{s}}$ is greater than $1100 \mathrm{~m} / \mathrm{s}$, they usually talk about "rigid" soil (no SSI effects - just rigid platform with a structure on it and excitation taken from the free field).

All these ranges are of course purely empirical. Obviously, one and the same soil can behave as rigid one towards very light and small structure (like a tent), and behave as a soft one towards heavy and rigid structure (like the NPP reactor building).

Sometimes to decide whether to account for SSI effects they compare the natural frequencies of the rigid structure on the flexible soil foundation with those of the flexible structure on a rigid foundation. If the lowest natural frequency of the first set is greater than the first dominant frequency of the second set two and more times, they do not consider SSI effects (e.g., see standards ASCE4-98 [1]). As SSI field is rather sophisticated, sometimes it is worth neglecting SSI, when allowed.

However, one should keep in mind another situation, when SSI effects occur. In soft soils seismic wave may have moderate wavelength comparable to the size of structure, so that 
the free-field motion over the soil-structure contact surface will have the so-called "space variability". In this case a comparatively stiff structure (even a weightless one) can impact the soil motion by structural rigidity (i.e., stiff contact soil-structure surface will not allow soil to move in the manner it used to move without structure). So, once more the presence of a structure changes the behavior of soil during seismic excitation, which is a SSI effect. This is a situation with considerably embedded structures; the same applies to the great base mats, "averaging" the travelling seismic waves [2]. But the most typical situation is a simple pile foundation - piles are used with soft soils, they are not rigid but usually stiff enough to change the foundation motion.

To conclude this part, let us talk a little about the so-called SSSI - structure-soil-structure interaction. When a group of structures is resting on a common soil foundation, the base mat motion of a structure may be changed (as compared to the free-filed motion) not because of this very structure only, but because of the neighboring structures. Of course, if all structures are comparatively light and no SSI effects occur for each of them standing alone, usually no SSSI effects occur for the group. But in case at least one of the structures standing alone can cause SSI effects, the additional waves in the soil (i.e. additional to the free-field wave picture) will spread from this structure, impacting neighbors. Sometimes (for the light/small neighbors) this situation can be analyzed without SSI but with special seismic excitation, accounting for the influence of the heavy neighbor. In other cases several structures should be analyzed together because of the mutual influence.

Thus, general goal of the SSI analysis is to calculate seismic response of structure based on seismic response of free field (sometimes free field may differ from the final soil). General format of the SSI analyses results is basements' motion obtained using the information about a) soil foundation (sometimes the initial one and the final one separately), b) structure, c) seismic excitation provided without structures. Another format of the SSI results is soilstructure interaction forces, necessary to estimate the soil foundation capability to withstand earthquake. Sometimes, the complete structural seismic response is obtained together with SSI in the format of response motion of different structural nodes and response structural internal seismic forces.

\section{Brief history}

As SSI field combines structures' and soil modeling, the level of such modeling is generally lower than in the classical soil mechanics and in the structural mechanics standing alone.

For several decades (up to 1960-s) only soil flexibility was considered without soil inertia springs modeled soil. At that time mostly the machinery basements were analyzed for the dynamic interaction with soil foundation (the largest of them probably being turbines). In fact, it was a quasi-static approach - the well-known static solution for rigid stamps, beams and plates on elastic foundation was applied at every time step. The model was so simple, that nobody even used the special term "SSI" at that time. The key question of such a simple approach appeared to be damping. The material damping measured in the laboratories with 
soil samples proved to be considerably less than the damping measured in the dynamic field tests with rigid stamps resting on the soil surface.

The nature of this effect was discovered in 1930-40s (Reissner in Germany [3], Schechter in the USSR) and proved to be in inertial properties of the soil. Inertia plus flexibility always mean wave propagation. It turned out that in the field tests actual energy dissipation in the soil was composed of two parts: conventional "material" damping (the same as in laboratory tests) and so-called "wave damping". In the latter case the moving stamp caused certain waves in the soil, and those waves took away energy from the stamp, contributing to the overall "damping" in the soil-structure system. This energy was not transferred from mechanical form into heat (like in material damping case), but was taken to the infinity in the original mechanical form. In reality waves did not go to the infinity, gradually dissipating due to the material damping in the soil, but huge volumes of the soil were involved in this wave propagation. Even without any material damping in the soil this "wave damping" contributed a lot to the response of the stamp. In practice it turned out that the level of wave damping was usually greater than the level of material damping.

In parallel it turned out that when the base mat size is comparable to the wave length in the soil, not only damping, but stiffness also depends on the excitation frequency. This effect is invisible for comparatively small machine basements, but important for large and stiff structures.

To study these wave effects new soil-structure models with infinite inertial soil foundations should be considered. That was the moment (1960-s) when the very term "SSI" appeared $[2,4,5]$. It happened so, that NPPs were actively designed at that time, including seismic regions (e.g., California), and intensive research was funded in the US to study the SSI effects controlling the NPP seismic response. Earthquake Engineering Research Center (EERC) in the University of Berkley, California became a leader with such outstanding scientists as H.B.Seed and J.Lysmer on board [6].

The main result of these investigations was the development of new powerful tools to analyze more or less realistic models. The earlier SSI models considered homogeneous halfspace with surface rigid stamp. They could be treated analytically or semi-analytically for simple stamp shapes (e.g., circle).

In practice soil is usually layered. Layering can lead to the appearance of the new wave types and change the whole wave picture. Important achievements of 1960-70s enabled to move from the homogeneous half-space to the horizontally-layered medium in soil modeling [7,8]. However, the infinite part of the foundation, excluding some limited soil volume around the basement, still remains a) linear, b) isotropic, c) horizontally layered. These limitations are due to the methods of the SSI analysis. The final masterpiece of Prof. Lysmer was SASSI code [9], further developed by F.Ostadan, M.Tabatabaie, D.Giocel et al. This code combined finite element modeling of the structure and limited volume of the soil with semi-analytical modeling of the infinite foundation (see below). Limitations on the embedment depth and on the shape of the underground part have gone. 
Some other scientists have greatly contributed to this field approximately at the same time (J.Wolf [10,11], J.M..Roesset, E.Kausel [12] and J.Luco [4] should be mentioned).

After the Three Miles Island and Chernobyl accidents there was a long pause in the nuclear energy development in the West (e.g., in 2012 US NRC issued a first permission for a new NPP block in last 33 years) and in Russia (due to the economic problems of 1990s), though in Asia they continued to build new blocks. SSI investigations went forward in South Korea, China, India. Nowadays, in spite of the Fukushima accident, nuclear industry goes forward.

In parallel SSI was studied by hydro-engineers (for the dams design, first of all). However, this field separated from the "NPP field of SSI" about 40 years ago. The reason was that the SSI models usually applied in nuclear industry (horizontally layered soil, rigid or very stiff base mats) are often not applicable to the hydro-dams situations (rocky canyons, etc.). That is why both models and methods used in the SSI field usually are different in nuclear and hydro-industries.

In last decades, civil structures are gradually increasing in size and embedment. Effects like SSI and SSSI from time to time are considered during the design procedures of such structures. Naturally, they are closer to the NPP practice than to the hydro-energy practice.

The author has about 30 years of experience in nuclear industry, dealing with SSI problems. So, the subsequent text will be based on the "NPP" approach to the SSI problems.

\section{Basic superposition}

Today there exist three approaches to SSI problems, namely "direct", "impedance", and "combined" ones. To understand them all, let us start from common general approach based on the superposition of the wave fields.

Let us call the problem with seismic wave, soil and structure "problem A" and start with completely linear soil-structure model. Let $\mathrm{Q}$ be some surface surrounding the basement in the soil and dividing the soil-structure model into two parts: the "external" part Vext and internal part $V_{\text {int. }}$ Let $(-F)$ be additional external loads distributed over $V_{\text {int }}$ and specially

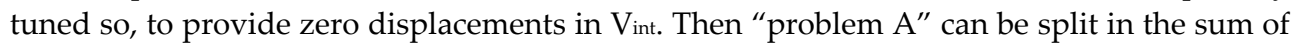
two wave pictures: "problem $\mathrm{A}_{1}$ ", including seismic excitation and loads $(-F)$, and "problem $\mathrm{A}_{2}$ " including only loads $(F)$ without seismic wave - see Fig.1.

This simple superposition leads to a number of important conclusions.

1. As in "problem $A_{1}$ " all displacements in the internal volume $V_{\text {int }}$ are zero, the motion of $V_{\text {int }}$ in "problem $\mathrm{A}_{2}$ " is the same as in "problem $A^{\prime}$. Hence, if we are interested in the

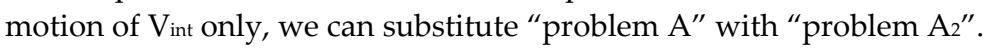

2. As in "problem $A_{1}$ " all displacements in the internal volume $V_{\text {int }}$ are zero, all the strains and internal forces in the internal volume $V_{\text {int }}$ are zero, and the external loads $(-F)$ must be zero everywhere in $V_{\text {int, }}$ except surface $Q$.

3. As in "problem $\mathrm{A}_{1}$ " all displacements, strains and internal forces in the internal volume $V_{\text {int }}$ are zero, no forces are impacting $Q$ from $V_{\text {int }}$ (i.e., forces impacting $Q$ from $V_{\text {ext }}$ are 
balanced by loads $(-F)$ ). Hence, $V_{\text {int }}$ can be withdrawn or replaced by another medium

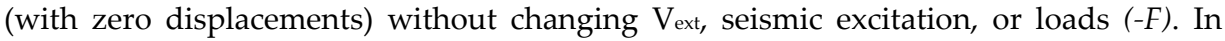
particular, $V_{\text {int }}$ can be replaced by initial soil without structure.

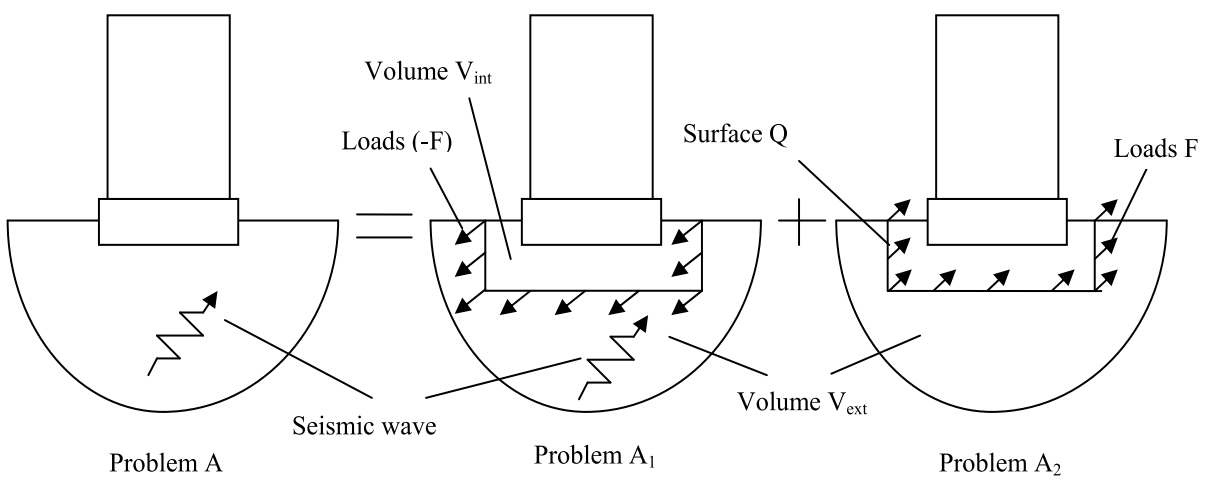

Figure 1. Basic superposition: split of the "problem $\mathrm{A}^{\text {" }}$

4. We can withdraw structure from Fig.1 and call the problem with initial soil in $V_{\text {int }}$ "problem B". This problem can be also split in "problem $\mathrm{B}_{1}$ " and "problem $\mathrm{B}_{2}$ " in the

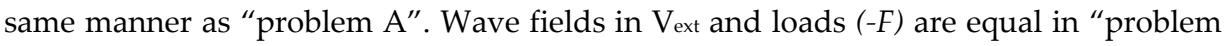
$\mathrm{A}_{1}$ " and "problem $\mathrm{B}_{1}$ ". However, in "problem $\mathrm{A}_{2}$ " and "problem $\mathrm{B}_{2}$ " wave fields are different in spite of similar loads $F$ and similar $V_{\text {ext }}$ in both problems. Generally, the motions of $\mathrm{Q}$ in "problem $\mathrm{B}_{2}$ " and "problem $\mathrm{A}_{2}$ " are different due to the waves, radiating from the structure in "problem $\mathrm{A}_{2}$ ".

5. Wave field in volume $\mathrm{V}_{\text {int }}$ in "problem $\mathrm{B}_{2}$ " is the same as in "problem B" (see conclusion 1 above). Very often this field is known apriori or easily calculated. This creates a powerful tool to verify models suggested for "problem $\mathrm{A}_{2}$ ". Each of these models contains some description of the internal part $V_{\text {int, }}$ external part $V_{\text {ext }}$ and the loads $F$. It is useful to take the same $V_{\text {ext }}$ and $F$ and substitute the internal part $V_{\text {int }}$ by the initial soil, thus coming from "problem $\mathrm{A}_{2}$ " to "problem $\mathrm{B}_{2}$ ". The suggested $V_{\text {ext }}$ and $F$ must provide adequate solution for "problem $\mathrm{B}_{2}$ "; otherwise they cannot be applied to "problem $\mathrm{A}_{2}$ ".

6. Loads $(-F)$ can be obtained from the wave field $U_{0}$ in "problem $B$ " and dynamic stiffness operator $G_{0}$ for the initial unbounded soil as follows

$$
-F(x)=G_{0}(x, y)\left[-U_{0}(y)\right]
$$

Formula (1) uses operator $G_{0}$ in the time domain. This operator is applied to the displacement field in the volume $V_{\text {int }}$ and provides the loads, distributed over the volume (this formula can be applied to the whole volume $V_{\text {int, }}$ but for the internal nodes the result will be zero). For linear initial soil this operator in the frequency domain will turn into complex frequency-dependent dynamic stiffness function. Note that for the given surface $Q$ the loads $F$ in (1) can be split in two parts: loads Fint acting from Vint and loads $F_{\text {ext }}$ acting from Vext. Physical meaning is illustrated in Fig.2. 


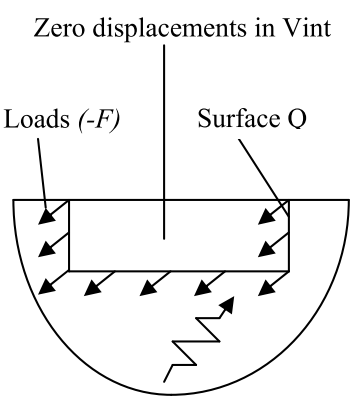

Problem $\mathrm{B}_{1}$

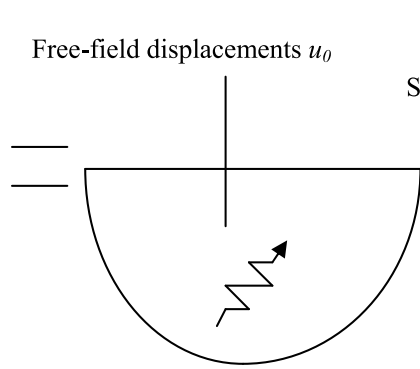

Problem B
Displacements $\left(-u_{0}\right)$

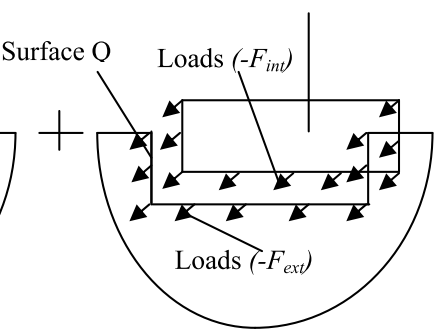

Reflected seismic wave

Figure 2. Split of loads $F$ into Fint and Fext

With given free-field wave field $U_{0}$ one can easily obtain $F_{\text {int }}$ just as surface forces at $Q$ corresponding to the internal stress field.

Theoretically we can change the content of the volume $V_{\text {int }}$ in "problem B" (e.g., withdraw the medium completely, taking loads $F_{\text {int }}$ to zero). This change will change operator $G_{0}$, change wave field $U_{0}$, but the left part of (1) will stay the same, as it is in fact fully determined by soil properties in $V_{\text {ext }}$ and initial seismic wave.

Even if there exists some physical non-linearity in the model, and this non-linearity is

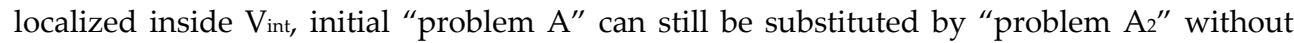
changing the loads $F$, as compared to the linear case. This is a consequence of the logic of the previous point: the loads are fully determined by soil in $V_{\text {ext }}$ and initial seismic wave.

\section{Classification of methods: direct and impedance approaches}

Current methods of SSI analysis can be classified according to the choice of surface Q. In "direct" method they try to put Q on some physical boundaries in the soil, usually apart from the basement. In "impedance" method they put Q right on the soil-structure contact surface, additionally presuming the rigidity of this surface. In "combined" method they put $\mathrm{Q}$ on the boundary of the "modified volume" (sometimes $\mathrm{Q}$ is a flexible soil-structure contact surface, but sometimes there exists some additional soil volume around the basement modified with the appearance of structure). Let us discuss some details of these methods one by one.

\subsection{Direct approach}

In direct method there always exists certain volume $\mathrm{V}_{\text {int. }}$ Usually the lower boundary (i.e., the bottom of $V_{\text {int }}$ ) is placed on the rock. In this case we presume that the additional waves radiating from the structure cannot change the motion of this boundary, as compared to the seismic field $U_{0}$. As a consequence, we can substitute boundary conditions in 
"problem $\mathrm{A}_{2}$ ", fixing the motion of the bottom (and obtaining it from "problem B") instead of modeling $V_{\text {ext }}$ and applying loads $F$ at surface $Q$. This substitution of the boundary conditions is shown in Fig.3.

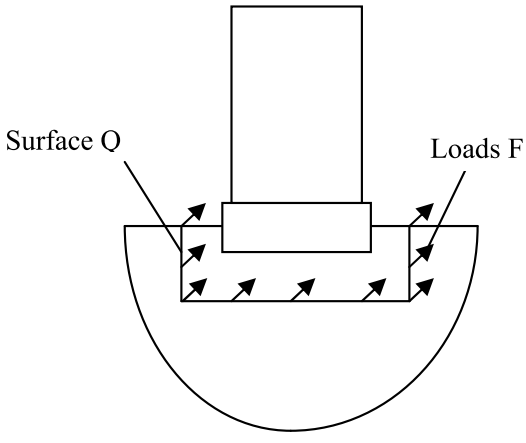

Problem $\mathrm{A}_{2}$

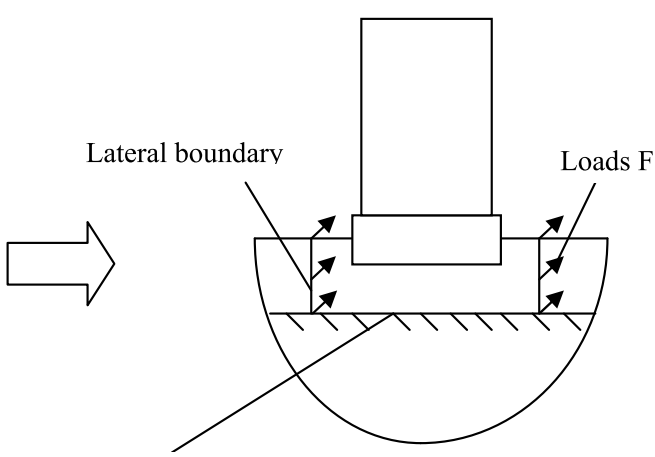

Lower boundary with prescribed motion $u_{0}$

Figure 3. Substitution of the boundary conditions at the bottom of $V_{\text {int }}$ in direct method

One should remember that such substitution is accurate for rigid rock only. Otherwise, an error occurs due to the reflection of the additional waves from such a boundary back into

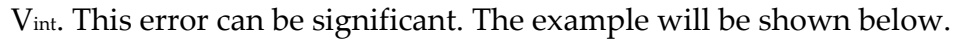

After the lower boundary is fixed, lateral boundaries (usually vertical) remain to be set. In 1970s, when direct method was popular, there was an evolution of these boundaries from "elementary" ones (fixed or free) towards more accurate ones. The first important step was so-called "acoustic boundaries" by Lysmer and Kuhlemeyer [13,14].

Physical base is as follows. 1D elastic (without material damping!) massive rod with shear or pressure waves can be cut in two, and one half of it can be accurately replaced by viscous dashpot. This is illustrated by Fig. 4 .

Dashpot viscosity parameter is a product of mass density $\rho$ and wave velocity $c$. So, Lysmer and Kuhlemeyer suggested to place distributed "shear" and "pressure" dashpots over vertical lateral boundary (three dashpots along three axes in each node). Practically they substituted the half-infinite layer in Fig.3 by number of horizontal 1D rods normal to the boundary.

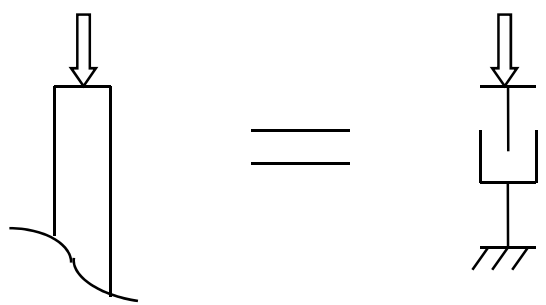

Figure 4. Analogue between half-infinite rod of unit cross-section area and viscous dashpot with viscosity parameter $b=\mathrm{Qc}$ 
This boundary was far better than any elementary (fixed or free) boundary before. Horizontal body waves normal to the boundary did not have artificial reflection into $V_{\text {int }}$ (that is why this boundary was called "non-reflecting boundary"). This boundary could work in the time domain. Besides, this boundary was "local": the response force acting to the node depended on the velocities of this node only, not neighbors.

This boundary was implemented in code LUSH [15], named after the authors (Lysmer, Udaka, Seed, Hwang). However, soon it turned out that the most important waves, radiating by structure in the soil underlain by rock are not horizontal body waves, but surface waves (see below). So, the artificial reflection at the boundary was not completely eliminated, and such a boundary could be placed only apart from the structure (3-4 plan sizes from the basements) to get more or less reasonable results.

One should keep in mind the important limitation: when wave process in $V_{\text {int }}$ is modeled by ordinary finite elements, the element size should be about $1 / 8$ (1/5 at most [1]) of the wavelength. It means that one cannot increase the finite element size going away from the structure (as they often do in statics). So, the increase in $V_{\text {int }}$ leads to the considerable increase in the problem size and to the computational problems.

The next step was done by G.Waas $[8,16,17]$. Instead of replacing the infinite soil by number of horizontal rods he suggested to use "homogeneous solutions" - i.e. the waves in the horizontally-layered medium underlain by rigid rock. These waves can be evaluated in the frequency domain only. According to G.Waas, in 2D case each homogeneous displacement varies in the horizontal direction following complex exponential rule with a certain complex frequency-dependent "wave number"; in the vertical direction it varies according to the finite-element interpolation. Later it turned out that in 3D case cylindrical waves in $V_{\text {ext }}$ along horizontal radius followed not exponents, but certain special Hankel functions with the same complex "wave numbers" as previously exponents in 2D case.

Any displacement of the lateral boundary (in the finite-element approximation) can be split into the sum of such waves in $V_{\text {ext. }}$ Then stresses are calculated at the boundary and finally nodal forces impacting $V_{\text {int }}$ from $V_{\text {ext }}$ in response to boundary displacement can be evaluated. So, the final format of the Waas boundary was the dynamic stiffness matrix in the frequency domain, replacing $V_{e x t}$ in the model. Unlike previous boundaries, this boundary was not local: response forces from $V_{\text {ext }}$ in the node depended not only on the motion of this very node, but on the motion of all the nodes. Thus, dynamic stiffness matrix became fully populated.

New boundaries (they were called "transmitting boundaries") proved to be far more accurate than previous ones. They could be placed right near the basement, decreasing $\mathrm{V}_{\text {int }}$ and accelerating the analysis. So, LUSH was converted into FLUSH (=Fast LUSH) [18] for 2D problems. Then appeared code ALUSH (=Axisymmetric LUSH) [19] to solve 3D problems with axisymmetric geometry. However, there remained two important limitations: hard rock at the bottom and axisymmetric geometry of structure in $3 \mathrm{D}$ case. The next step forward was again done by J.Lysmer in EERC. But this new approach will be discussed later (see "combined method"). 


\subsection{Impedance approach}

If we presume the rigidity of the soil-structure contact surface and place surface $Q$ in Fig.1 right at this surface, we get six degrees of freedom for $Q$. Corresponding forces $F$ in "problem $\mathrm{A}_{2}$ " are condensed to six-component integral forces, loading the immovable base mat during seismic excitation. In addition, when the mat moves, $V_{\text {ext }}$ impacts the moving base mat by response forces set by "dynamic stiffness matrix" $6 \times 6$. This matrix is a linear operators' matrix in the time domain for linear soil (soil in Vext was linear from the very beginning). In the frequency domain it becomes complex frequency dependent matrix called "impedance matrix". That is why the whole approach is called the "impedance" one. Impedances can be estimated in the field experiments with stamps excited by unbalanced rotors. They were the first parameters of the soil flexibility in the early times of SSI (when machinery base mats were analyzed).

Let $R_{j}(x)$ be real "rigid" vector displacements field of the contact surface $\mathrm{Q}$, when one of six general coordinates of the surface $\mathrm{Q}$ (coordinate number $j$ ) gets a unit displacement. If some contact vector forces $F(x)$ act over $\mathrm{Q}$, the condensed integral general force along coordinate $j$ is given by

$$
P_{j}=\int_{Q} R_{j}^{T}(x) F(x) d Q
$$

Scalar product of two 3D vectors is used under the integral (2). If $G_{0}$ is the dynamic stiffness operator in the initial soil, and $\mathrm{Q}$ moves by unit along the general coordinate $k$, then the displacement field at $\mathrm{Q}$ is described by $R_{k}(x)$, and response forces impacting $\mathrm{Q}$ in the initial soil are given by $G_{0} R_{k}$. However, for the embedded $Q$ these total forces consist of the part $F_{k}(x)$ impacting $\mathrm{Q}$ from $\mathrm{V}_{\text {ext }}$ and another part impacting $\mathrm{Q}$ from $\mathrm{V}_{\text {int }}$ (see Fig.2). If Gint is the analogue of $G_{0}$ for the finite volume $V_{\text {int, then }} F_{k}=\left(G_{0}-G_{i n t}\right) R_{k}$. In the frequency domain all linear operators become matrices. The impedance $C_{j k}$, meaning the condensed force acting from $\mathrm{V}_{\text {ext }}$ to $\mathrm{Q}$ along coordinate $j$ in response to the unit rigid displacement of $\mathrm{Q}$ along coordinate $k$ is then given by

$$
C_{j k}(\omega)=\int_{Q_{x}} \int_{Q_{y}} R_{j}^{T}(x)\left[G_{0}(\omega)-G_{\text {int }}(\omega)\right] R_{k}(y) d Q_{x} d Q_{y}
$$

Double integration in (3) reflects the fact that $G$ provides the response forces in the node $x$ due to the displacements in the node y.

The next step is to condense load $F$ over rigid surface Q. If $U_{k}(x)$ is a transfer function from the control motion along coordinate $k$ to the free-field wave in "problem B", then in the frequency domain the transfer function $B_{j k}$ to the condensed load along coordinate $j$ according to (1) is given by

$$
B_{j k}(\omega)=\int_{V_{x}} \int_{V_{y}} R_{j}^{T}(x)\left[G_{0}(\omega)\right] U_{k}(y) d V_{x} d V_{y}
$$


If $U_{0}$ is a control motion of a certain point in "problem B" (generally multi-component one, though usually only three-component one), then the condensed total forces (not just F!) acting from the soil to the basement are given by

$$
P(\omega)=B(\omega) U_{0}(\omega)-C(\omega) U_{b}(\omega)
$$

Here $U_{b}(\omega)$ is a six-component motion of the rigid base mat (i.e., soil-structure contact surface $Q), C(\omega)$ is $6 \times 6$ impedance matrix, $B(\omega)$ is usually $6 \times 3$ seismic loading matrix.

The important particular case is a surface basement - then $V_{\text {int }}$ goes and Gint in right-hand part of (3) goes as well. If in addition we presume $U_{k}(y)=R_{k}(y)=1, k=1,2,3$ (it means that the whole future contact surface $Q$ in "problem B" has no seismic rotations and has the same translations as the control point; this is the case for vertically propagating seismic body waves in the horizontally-layered media), then matrix $B$ is composed of the first three columns of matrix $C$.

Let $K(\omega)$ and $M$ be stiffness and mass matrices of the structure without soil foundation ( $K$ may be complex to account for the structural internal damping), and let $U_{s}(\omega)$ be absolute displacements of structure in the frequency domain. Then seismic motion in the soilstructure system is described by the equation

$$
\left[K(\omega)-\omega^{2} M\right] U_{s}(\omega)=P(\omega)
$$

Matrix $\left[K-\omega^{2} M\right]$ is huge in size. Loads $P(\omega)$ are non-zero only for the rigid basement's six degrees of freedom. As displacements $U_{b}$ take part in (5), and they are at the same time part of $U_{s}$, they should better join the left-hand part of (6), making (6) look like

$$
\left[K(\omega)-\omega^{2} M+C(\omega)\right] U_{s}(\omega)=B(\omega) U_{0}(\omega)
$$

In (7) $C(\omega)$ has to be huge matrix of the same size as $K$ and $M$, but only $6 \times 6$ block of it referring to $U_{b}$ is populated.

Now let us imagine the same rigid contact surface on the same soil with the same seismic excitation, with the only difference: structure with rigid basement is weightless. The righthand part of (7) will stay in place; in the left-hand part only $C(\omega)$ represents dynamic stiffness, because weightless structure is moving rigidly together with the basement, and no forces occur due to $K$. Let $V_{b}(\omega)$ be the response of the rigid weightless basement (instead of $U_{b}(\omega)$ for massive structure:

$$
C(\omega) V_{b}(\omega)=B(\omega) U_{0}(\omega)
$$

Using (8) one can replace the right-hand part of (7) and come to the equation

$$
\left[K(\omega)-\omega^{2} M+C(\omega)\right] U_{s}(\omega)=C(\omega) V_{b}(\omega)
$$

This equation is of great importance because it describes the so-called "platform" model, shown in Fig.5. Rigid platform is excited by a prescribed motion $V_{b}(\omega)$. Structural model is resting on a "soil support" with prescribed stiffness described by the impedance matrix $C(\omega)$. 

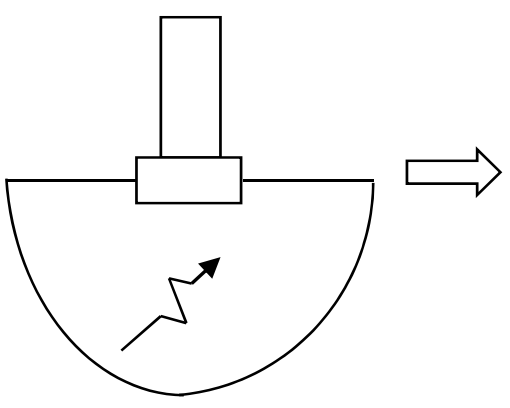

Weightless rigid basement with seismic response $V_{b}$

Figure 5. Scheme of platform model for SSI analysis
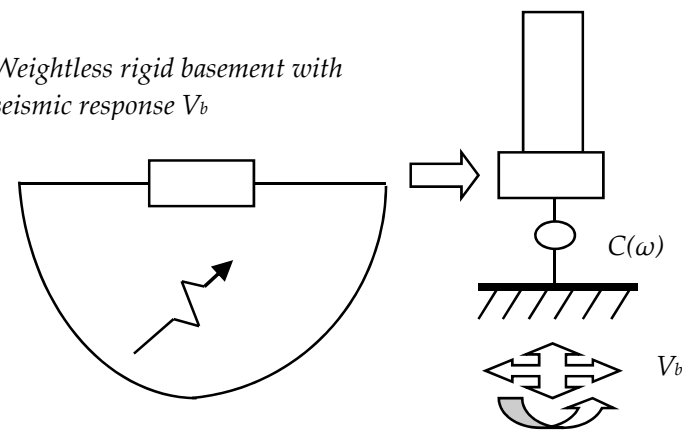

One should clearly understand that this platform is not physical: in the real world there is no rigid platform having such a motion. Often they try to imagine some hard rock somewhere - there is a mistake! This model is applicable, for example, for homogeneous half-space - no rock is present anywhere. In fact, platform model is just a mechanical analogue of the soil-structure model in terms of the structural response.

Let us work a little with this platform model. In the popular particular case with two assumptions mentioned above (i.e. surface rigid basement and vertical seismic waves in the horizontally layered medium) weightless rigid base mat will move exactly with "control" accelerations from free field. In other words $V_{b}=U_{0}$. This case is shown in Fig.6.
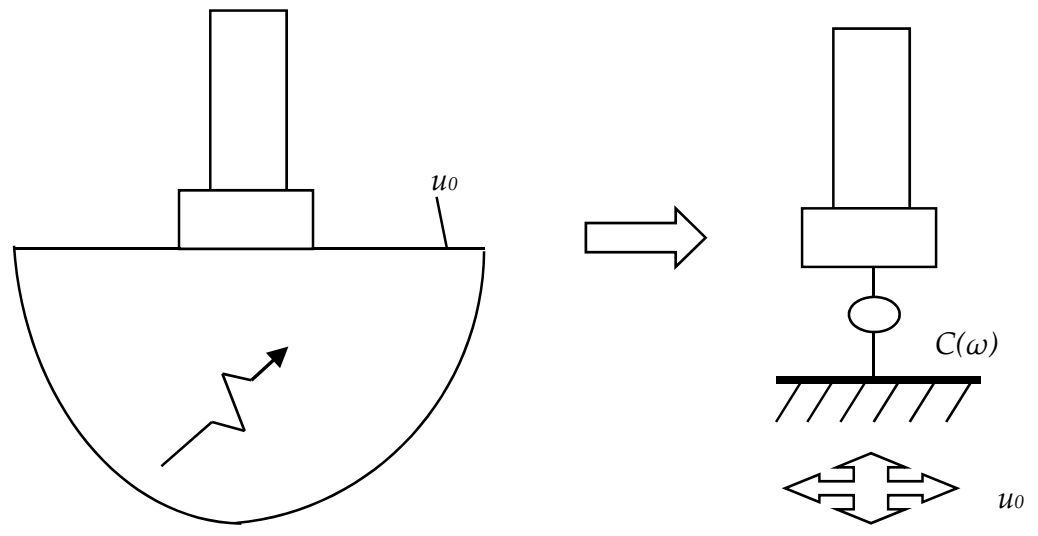

Figure 6. Scheme of platform model for SSI analysis with two assumptions: rigid surface basement and "rigid" free-field motion under it

If any of the two assumptions is not valid, one has to solve a special problem to obtain $V_{b}-$ it is called "kinematic soil-structure interaction problem". For embedded basements it is allowed to neglect the embedment depth if it is less then $30 \%$ of the effective radius of the mat. In this case upper layers of the soil foundation are just withdrawn from the soil model. This may sometimes cause some changes in the free-field motion. 
However, even for the surface basements the second assumption is not always correct. If the free-field motion $U_{0}$ of the soil surface is not "rigid" over $Q$, the rigid basement's response is somehow averaging it over the mat; in this case the translational response $V_{b}$ may be less than control motion $U_{0}$ in a single node. On the other hand, the variability of horizontal $U_{0}$ under the mat may cause torsion in $V_{b}$, and the variability of vertical $U_{0}$ under the mat may cause rocking in $V_{b}$.

The second part of the problem (i.e. the dynamic analysis of the platform model from Fig.5) is called "inertial soil-structure interaction problem". Often they solve it putting the coordinate system on the platform (thus, coming to the fixed platform, but instead introducing inertial forces impacting all the masses of structure).

One can easily see that conventional structural seismic response without SSI is obtained from the platform model as well. The difference with Fig.5 is only in the soil support and in the platform excitation. If we make soil stiffer and stiffer, $V_{b}$ comes closer and closer to $U_{0}$; "soil support" becomes stiff, and this difference also goes. So, we get a model without SSI as a limit case of the platform model with SSI with extremely hard soil.

Platform model can be extended to the case of several buildings having common soil foundation. The main limitations are the same - rigid basements and linear foundation/contact. Note that generally (with "kinematic interaction" on Fig.5) no requirements are made for soil layering, embedment depth, shape of the underground part, etc.

The alternative form of the platform model uses fixed platform, and dynamic loads are directly applied to the rigid basement. These loads correspond to the right-hand parts of (9), (8) or (7).

Platform model is convenient to work with in the frequency domain. Huge matrix $\left[K-\omega^{2} M\right]$ can be condensed to the rigid base mat using natural modes and natural frequencies of the fixed-base structure [20]. As a result, we get $M(\omega)$ - the "dynamic inertia" complex frequency-dependent matrix $6 \times 6-$ similar in size to $C(\omega)$. Equation (7) turns to

$$
\left[C(\omega)-\omega^{2} M(\omega)\right] U_{b}(\omega)=B(\omega) U_{0}(\omega)
$$

It is easily solved, because maximal size is 6 . The response in the time domain is further obtained using Fast Fourier Transform (FFT). For multiple-base structure the dynamic stiffness is condensed in a somewhat different way [21], though the size of the resulting matrix is still $6 \times$ (number of base mats).

However, usually engineers prefer the time domain for the dynamic problems. Platform model of Fig.5 and equation (9) can be transferred to the time domain. Structural part is transferred easily. Kinematic excitation $V_{b}$ can be also transferred to the time domain using FFT. The only problem is to transfer to the time domain impedance matrix $C(\omega)$.

The most popular variant is just a set of six springs and six viscous dashpots (one spring and one dashpot along each of six coordinates). In the frequency domain an ordinary spring 
corresponds to the real frequency-independent impedance, and viscous dashpot corresponds to the frequency-linear purely imaginary impedance. So, matrix $C(\omega)$ corresponding to such a set of springs/dashpots will be diagonal complex matrix $6 \times 6$ with frequency-independent real parts and frequency-linear imaginary parts. Is it realistic for real-world soil foundations? This question deserves special discussion. But before we enter it, let us consider a very simple example, illustrating methodology of SSI problem as a whole and two basic different approaches to this problem (i.e., direct and impedance ones) described above.

\section{Sample 1D SSI problem as an example of different approaches to SSI analysis}

Let us consider 1D P-waves in a homogeneous massive rod, modeling soil. The only coordinate is $x$. Wave displacements $u(x, t)$ are described by the wave equation

$$
c^{2} \frac{\partial^{2} u}{\partial x^{2}}=\frac{\partial^{2} u}{\partial t^{2}}
$$

Here $c$ is wave velocity. In the frequency domain for certain frequency $\omega$ there exist two solutions of (11):

$$
u_{1}=U_{1} \exp (-i x / \lambda) ; \quad u_{2}=U_{2} \exp (i x / \lambda) ; \lambda=c / \omega
$$

The first wave $u_{1}$ described by (12) goes up along Ox, the second wave $u_{2}$ goes down. Each wave has own amplitude $U$. Wave velocity $c$ is determined by mass density $\rho$ and constrained elasticity module $E^{0}$ as

$$
c^{2}=E^{0} / \rho
$$

Let us now apply basic principles discussed above to this simple model: solve "problem B", "problem A", and then solve "problem $\mathrm{A}_{2}$ " by direct and impedance methods. All problems will be solved in the frequency domain, using (12).

\subsection{The exact solution}

Let us start with "problem B" - wave solution without structure. Let $U^{0}$ be displacement at the free surface $x=0$. As we are going to obtain coefficient $U_{1}{ }^{0}$ of the upcoming wave and coefficient $U_{2}{ }^{0}$ of the wave coming down, now we have the first of the two equations for them

$$
u^{0}(0)=U_{1}^{0}+U_{2}^{0}=U^{0}
$$

The second equation comes from the description of the "free" condition of the surface; total stress in the soil must be zero: 


$$
E^{0} \frac{\partial u^{0}}{\partial x}(0)=E^{0}(i / \lambda)\left(-U_{1}^{0}+U_{2}^{0}\right)=0
$$

Two equations (14) and (15) give us the well-known "doubling rule": the upcoming wave reaching free surface reflects back. At the free surface displacements of the upcoming wave are doubled:

$$
U_{1}^{0}=U^{0} / 2 ; \quad U_{2}^{0}=U^{0} / 2 ; \quad u^{0}(x)=U^{0}[\exp (-i x / \lambda)+\exp (i x / \lambda)] / 2=U^{0} \cos (x / \lambda)
$$

So, (16) gives the whole solution of the "problem B" linking wave field in any point to the "control motion" $U_{0}$.

Now let us move to the "problem A". Let structure be rigid and have mass $m$ (this is a mass, related to the unit area of the cross-section of the rod). Schemes of "problem B" and "problem A" are shown on Fig.7.

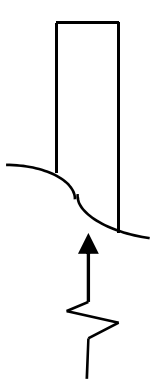

Problem $B$

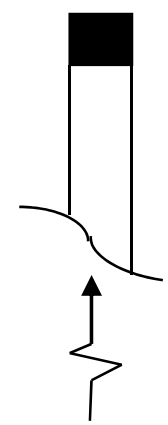

Problem A

Figure 7. Layouts of "problem B" and "problem A"

General goal of the SSI analysis is to obtain the response motion of the basement from the given control motion of the free surface. In this simple case we can do it directly (it is not the "direct approach" so far, just a simple solution!). In "problem A" wave field in the soil is still described by (12), but with coefficients $U^{A_{1}}$ (upcoming wave) and $U^{A_{2}}$ (wave coming down):

$$
u^{A}(x)=U_{1}^{A} \exp (-i x / \lambda)+U_{2}^{A} \exp (i x / \lambda)
$$

Equation of motion for mass $m$ in the frequency domain is

$$
-\omega^{2} m\left(U_{1}^{A}+U_{2}^{A}\right)=-E^{0}\left[(-i / \lambda) U_{1}^{A}+(i / \lambda) U_{2}^{A}\right]
$$

Having (18) one can express the reflected wave amplitude from the amplitude of the upcoming wave

$$
U_{2}^{A}=U_{1}^{A}\left[i E^{0} / \lambda+\omega^{2} m\right] /\left[i E^{0} / \lambda-\omega^{2} m\right]=U_{1}^{A}\left[1-i \frac{m}{\rho \lambda}\right] /\left[1+i \frac{m}{\rho \lambda}\right]
$$


"Problem A" and "problem B" have similar seismic excitation: in our case it means that the upcoming waves are similar:

$$
U_{1}^{A}=U_{1}^{0}=U^{0} / 2
$$

Hence we get the final expression linking the displacement of the mass in "problem $\mathrm{A}^{\text {" to }}$ the control motion at the free surface in "problem B":

$$
U^{A}=U^{0}\left\{1+\left[1-i \frac{m}{\rho \lambda}\right] /\left[1+i \frac{m}{\rho \lambda}\right]\right\} / 2=U^{0} /\left[1+i \frac{m \omega}{\rho c}\right]
$$

The transfer function in the frequency domain linking response displacements to the control displacements in (21) at the same time links response accelerations to the control accelerations. Having control accelerogram $a_{0}(t)$ one can get response accelerogram $a_{A}(t)$ easily using FFT technique.

\subsection{Direct approach}

Now we will show the direct approach for the same example, following p.4.1. Let surface $Q$ be at depth $H$. There the free-field wave according to (16) will be

$$
u^{0}(-H)=U^{0} \cos (H / \lambda) ; \quad \frac{\partial u^{0}}{\partial x}(-H)=\left(U^{0} / \lambda\right) \sin (H / \lambda)
$$

Loads (-F) may be obtained from "problem $\mathrm{B}_{1}$ " as shown on Fig.2. In the upper part the resulting sum must be zero; so, the part $F_{\text {int }}$ of the load, balancing the reflected wave in the upper part of the soil, is just "mirror" of the free field:

$$
F_{\text {int }}=-E^{0} \frac{\partial u^{0}}{\partial x}(-H)=-\left(E^{0} U^{0} / \lambda\right) \sin (H / \lambda)=-\rho \omega c U^{0} \sin (H / \lambda)
$$

The reflected wave $u_{1}$ in the lower part of the rod consists just of the single wave coming down. We know the displacement at Q; so, we can describe the additional wave in the lower part as

$$
u^{1}(x)=-U^{0} \cos (H / \lambda) \exp [i(x+H) / \lambda] ; \quad \frac{\partial u^{1}}{\partial x}(x)=-\left(i U^{0} / \lambda\right) \cos (H / \lambda) \exp [i(x+H) / \lambda]
$$

In this wave field at $x=-H$ we get the following force $F_{e x t}$, impacting $\mathrm{Q}$ from the lower part of the soil

$$
F_{\text {ext }}=E^{0}\left(i U^{0} / \lambda\right) \cos (H / \lambda)=i U^{0}\left(\rho c^{2} \omega / c\right) \cos (H / \lambda)=(i \omega \rho c) U^{0} \cos (H / \lambda)
$$

Thus, the total load $F$ in "problem $\mathrm{B}_{1}$ " is composed of two parts, given by (23) and (25):

$$
F=F_{\mathrm{int}}+F_{\text {ext }}=i \omega \rho c U^{0}[\cos (H / \lambda)+i \sin (H / \lambda)]=i \omega \rho c U^{0} \exp (i H / \lambda)
$$


The same load $F$ will impact $Q$ in "problem $\mathrm{B}_{2}$ " and in "problem $\mathrm{A}_{2}$ ". To complete the model we use the analogue between half-infinite rod and viscous dashpot, mentioned above (remember boundaries of Lysmer-Kuhlemeyer) and shown on Fig.4.

As a result, complete models for the direct approach look now like those shown on Fig.8
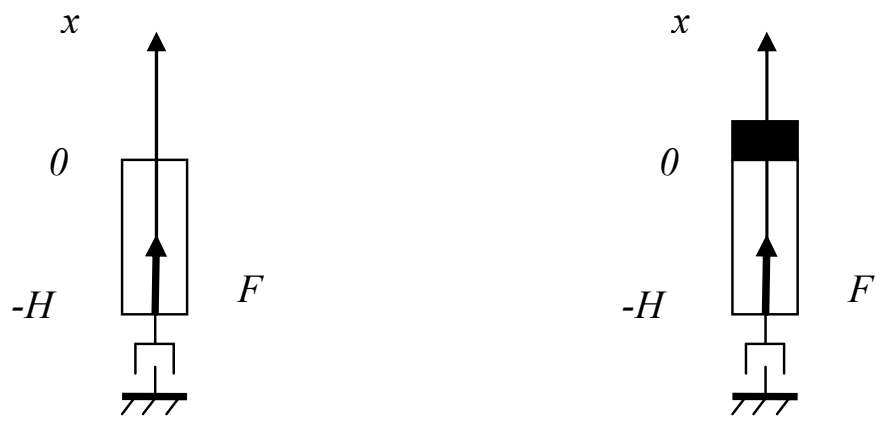

Figure 8. Direct approach models for "problem $\mathrm{B}_{2}$ " and "problem $\mathrm{A}_{2}$ "

As previously mentioned it is worth solving "problem $\mathrm{B}_{2}$ " first and compare the solution with free-field. Instead of that we can take "exact" displacement from exact wave field at $Q$, calculate total force impacting the upper part in the model on Fig.8 and check the equilibrium for $\mathrm{Q}$. In our case the force impacting the upper part from dashpot is a product of exact velocity $\left[i \omega U_{0}(-H)\right]$ and viscous parameter of dashpot $(\rho c)$; we see that it annihilates with $F_{\text {ext }}$ given by (25). The rest of the load $F$ - the load $F_{\text {int }}$ - annihilates with internal forces in the upper part due to the origin (23). So, "problem $\mathrm{B}_{2}$ " gives the exact solution.

Now let us check the equilibrium for $\mathrm{Q}$ in "problem $\mathrm{A}_{2}$ ". The exact solution is given by $(17,19,20)$ :

$$
U^{A}(x)=U^{0}\left\{\exp (-i x / \lambda)+\left[1-i \frac{m}{\rho \lambda}\right] /\left[1+i \frac{m}{\rho \lambda}\right] \exp (i x / \lambda)\right\} / 2
$$

So, $\mathrm{Q}$ is loaded by a) force from dashpot, $\mathrm{b}$ ) internal force, acting from the upper part (not $F_{\text {int }}$ any more, because in the upper part wave field has changed!) and c) the external load $F$ given by (26):

$$
-i \omega \rho c U^{A}(-H)+E^{0} \frac{\partial U^{A}}{\partial x}(-H)+F=0
$$

Let a reader obtain zero in (28) himself. General conclusion is that direct method provides exact results, if correctly applied.

Now let us perform the substitution of the boundary conditions in the direct approach, shown on Fig.3, i.e. let us fix the motion of $\mathrm{Q}$ as $U^{0}(-H)$. We see that "problem $\mathrm{B}_{2}$ " without 
structure will be solved exactly, providing $U^{0}(x)$ in the whole upper part. So, this check is OK. However, in "problem $\mathrm{A}_{2}$ " exact displacement $U^{A}(-H)$ given by (27) is different from $U^{0}(-H)$ given by (22). As a result, both upcoming wave and the wave coming down in $V_{\text {int }}$ will be different from the exact solution. If this new solution is marked with upper index " 3 ", then the displacement of the mass is given by

$$
U^{3}=U_{1}^{3}+U_{2}^{3}=U^{0} /\left[\cos (H / \lambda)-\frac{m}{\rho \lambda} \sin (H / \lambda)\right]
$$

Note that this solution depends on $H$, while the exact solution does not (see (21)). As our boundary is artificial, such dependence cannot be physical.

We can calculate the "error coefficient" relating approximate solution (29) to the exact solution (21). With dimensionless frequency

$$
\varpi=\frac{\omega m}{\rho c}=\frac{m}{\rho \lambda}
$$

and dimensionless depth of $\mathrm{Q}$

$$
h=H \frac{\rho}{m}
$$

this "error coefficient" makes

$$
\mu=\frac{U^{3}}{U^{A}}=\frac{1+i \varpi}{\cos (h \varpi)-\varpi \sin (h \varpi)}
$$

Curves for different $\mu(\omega)$ for different $h$ are shown in Fig.9.

We see that the solution in not satisfactory. The increase of the boundary depth $h$ does not improve the situation. General conclusion is that one must be very careful in placing lower boundary in the direct approach when there is no rock seen in the depth.

\subsection{Impedance approach}

Now let us apply the impedance approach to the same system. As our structure rests on the surface, we can use Fig.6. If the displacement of the mass is $U_{b}$, then the equation of motion (10) turns to

$$
\left[C(\omega)-\omega^{2} m\right] U_{b}(\omega)=C(\omega) U^{0}(\omega)
$$

Impedance is the same as in Fig.4: $C(\omega)=i \omega \rho c$. So, from (33) we at once get the ultimate result which turns to be similar to the exact one (21):

$$
U_{b}=U^{0} /\left[1-\frac{\omega m}{i \rho c}\right]=U^{0} /\left[1+i \frac{m}{\rho \lambda}\right]=U^{A}
$$




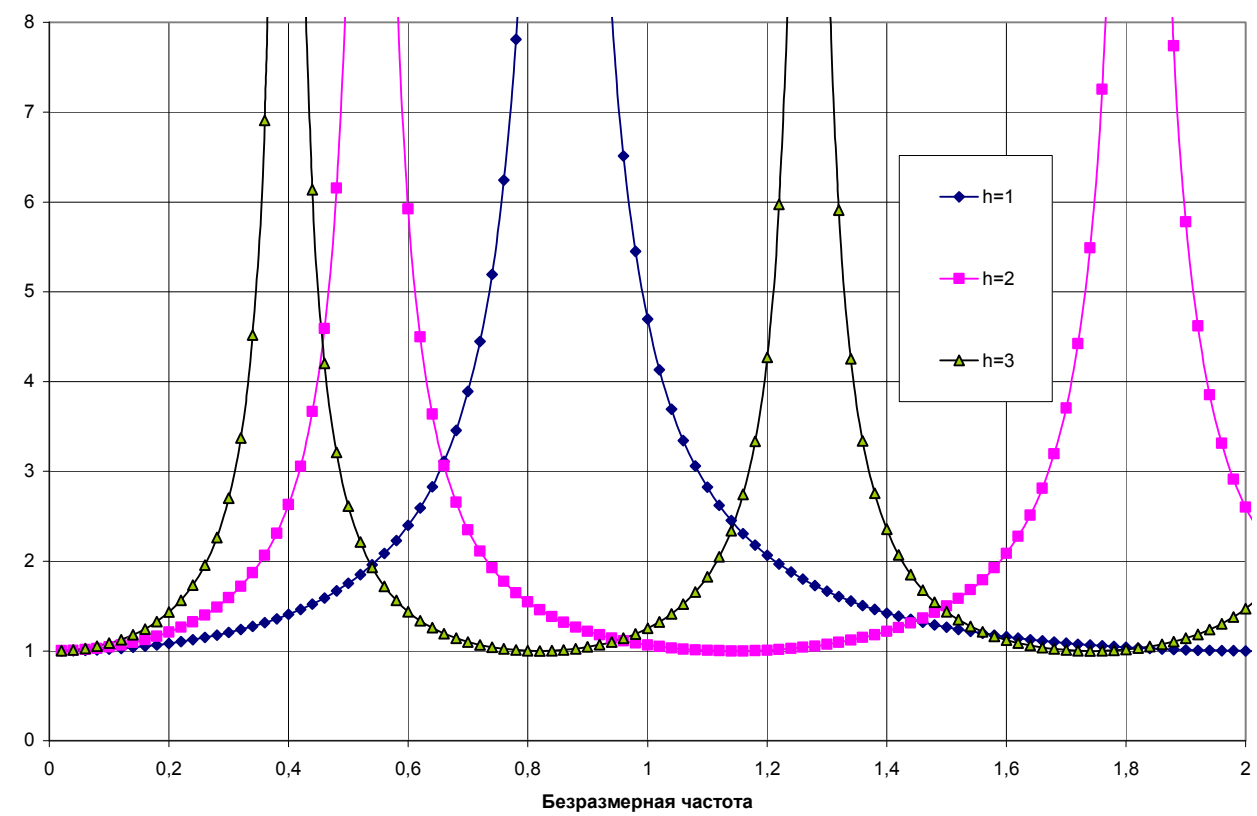

Figure 9. "Error coefficient" for direct approach after changing the lower boundary condition

General conclusion is that the correct application of the impedance method provides the exact solution.

The decisive factor in getting the exact solution both in direct and in impedance approaches for our 1D model was the ability to get exact model for substituting Vext (dashpot in our case).

Unfortunately, this example is of methodology value only. While "problem B" (without structure) is often treated in practice with 1D models (though not homogeneous), "problem $\mathrm{A}^{\prime \prime}$ (with structure) is principally different. Great part of energy is taken from the structure by surface waves, which are not represented in 1D model.

\section{Impedances in the frequency domain}

Historically, the first problems for impedances considering soil inertia were solved semianalytically for homogeneous half-space without the internal damping and for circular surface stamps. It turned out that horizontal impedances behaved more or less like pairs of springs and viscous dashpots (as mentioned above, in reality the dissipation of energy had completely different nature; mechanical energy was not converted in heat, as in dashpot, but taken away by elastic waves). One more "good news" was that the impedance matrix appeared to be almost diagonal. Though non-diagonal terms coupling horizontal translation with rocking in the same vertical plane were non-zero ones, their squares were considerably less in module than products of the corresponding diagonal terms of the impedance matrix. 
These two facts created a base for using the above mentioned "soil springs and dashpots". There are several variants of stiffness and dashpot parameters. You can see below a table 1 from ASCE4-98 [1] for circular base mats.

\begin{tabular}{|c|c|c|}
\hline Motion & Equivalent Spring Constant & Equivalent Damping Coefficient \\
\hline Horizontal & $k_{x}=\frac{32(1-v) G R}{7-8 v}$ & $c_{x}=0.576 k_{x} R \sqrt{\rho / G}$ \\
\hline Rocking & $k_{\psi}=\frac{8 G R^{3}}{3(1-v)}$ & $c_{\psi}=\frac{0.30}{1+B_{\psi}} k_{\psi} R \sqrt{\rho / G}$ \\
\hline Vertical & $k_{z}=\frac{4 G R}{(1-v)}$ & $c_{z}=0.85 k_{z} R \sqrt{\rho / G}$ \\
\hline Torsion & $k_{t}=16 G R^{3} / 3$ & $c_{t}=\frac{\sqrt{k_{t} I_{t}}}{1+2 I_{t} / \rho R^{3}}$ \\
\hline
\end{tabular}

Notes: $v=$ Poisson's ratio of foundation medium; $G=$ shear modulus of foundation medium; $R$ = radius of circular base mat; $\rho=$ mass density of foundation medium; $B_{\psi}=3(1-v) I_{0} / 8 \rho R^{5} ; I_{0}=$ total mass moment of inertia of structure and base mat about the rocking axis at the base; and $I_{t}=$ polar mass moment of inertia of structure and base mat.

Table 1. Lumped Representation of Structure-Foundation Interaction at Surface for Circular Base

One more table of the same sort in the same standard [1] is given for rectangular base mats.

However, even for homogeneous half-space it turned out that vertical and angular impedances behaved differently from simple springs and dashpots. This can be seen in Fig.10, where the tabular impedances given by ASCE4-98 are compared to the wave solutions given by codes SASSI and CLASSI (number in the legend denotes number of finite elements along the side).

We understand now that the expressions given in the tables are just approximations for the real values. So, there cannot be "exact" or "true" expressions of this kind - different variants exist.

The expressions from the tables look particularly strange for angular damping, where parameters of the upper structure participate. It is real absurd from the physical point of view: impedances cannot depend on the upper structure; soil just "does not know" what is above rigid stamp. This is not an error, as some colleagues think. This is an attempt to take values from the frequency-dependent curves at certain frequencies. These frequencies estimate the first natural frequencies of rigid structure on flexible soil, so they depend on structural inertial parameters. Surely, such expressions are approximate.

The conclusion is that frequency dependence of the impedances exists even for the homogeneous half-space and spoils the spring/dashpot models in the impedance method. 

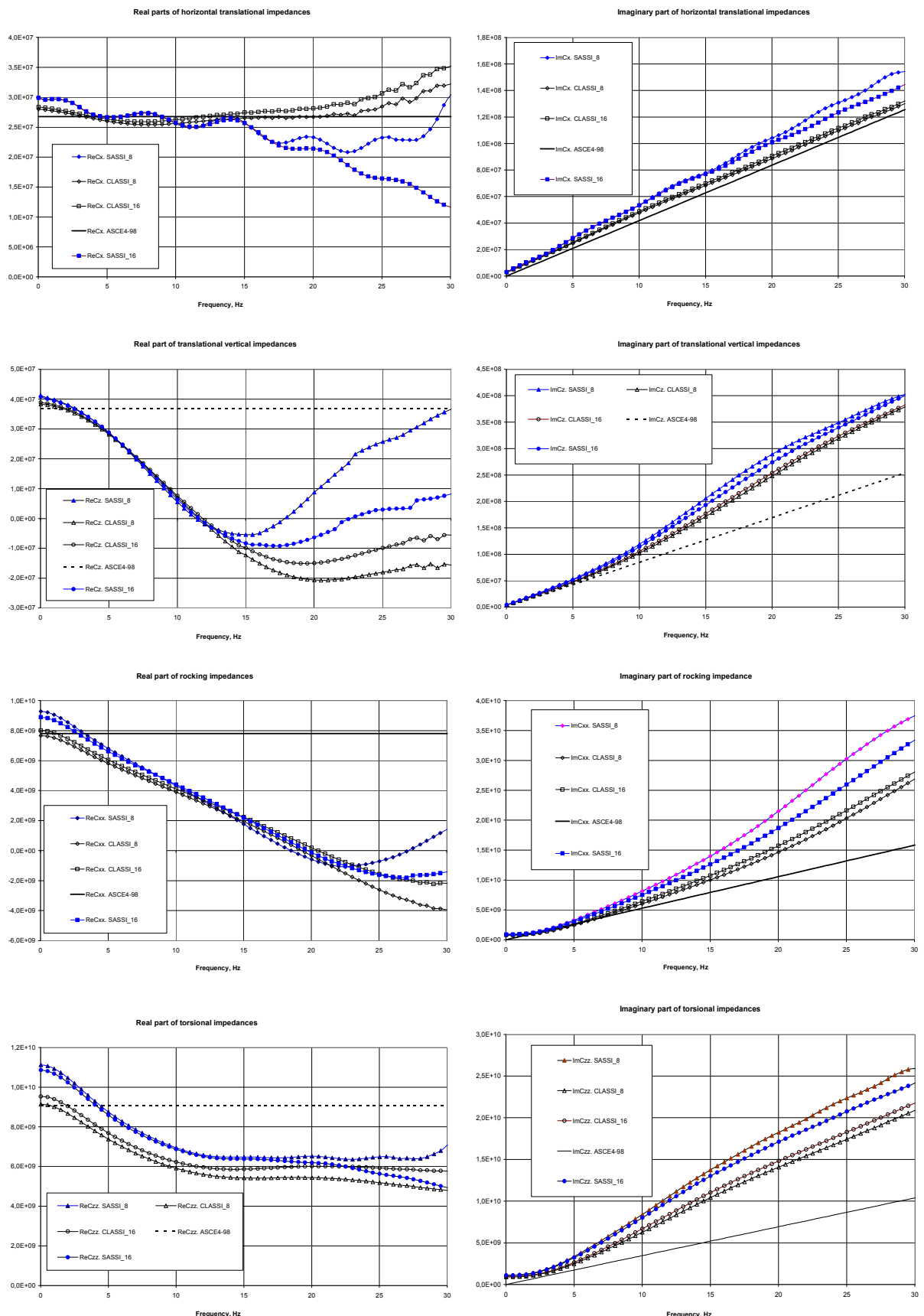

Figure 10. Impedances for a basement $30.6 \times 30.6$ meters on the surface of homogeneous half-space with mass density $\varrho=2 \mathrm{t} / \mathrm{m}^{3}$, wave velocities $\mathrm{V}_{\mathrm{s}}=400 \mathrm{~m} / \mathrm{s}, \mathrm{V}_{\mathrm{p}}=1100 \mathrm{~m} / \mathrm{s}$, internal damping $\gamma=5 \%$. 
One more comment should be added here. If a package of horizontal layers is underlain by rigid rock, surface waves behave in a completely different manner than for homogeneous half-space. Instead of two surface waves (Love and Rayleigh ones) there exist an infinite number of surface waves. Each of these waves for low frequencies cannot take energy from the basement - they are "geometrically dissipating" (even without internal soil damping), or "locked". However, when frequency goes up, each of these waves one by one transforms from a "locked" wave into a "running" wave capable to take energy to the infinity. This behavior depends on soil geometry only, not on structure.

It means that in the frequency domain there exists a certain low-frequency range, where the whole soil foundation is "locked". All the energy taken from the basement is only due to the internal damping. If there is no internal damping, complex impedances are completely real. In practice internal soil damping is several percent, so impedances are "almost real". After the first surface wave transforms into a running one, the soil foundation becomes "unlocked" - wave damping appears. Then one by one other surface waves turn into "running" ones, increasing the integral damping in the soil-structure system. The impedance functions for the same soil, but underlain by rigid rock at depth $26 \mathrm{~m}$, are shown on Fig.11. One can see "locking phenomena" looking at the imaginary parts of the impedances.

The conclusion here is that the frequency dependence of the impedances may be rather sophisticated depending on soil layering. The attempts to "cover" the variety of soils by number of homogeneous half-spaces with different properties (usually this is an approach for "serial design" of structures) may lead to mistakes: there is always a possibility that real layered soil will not be "covered" by a set of half-spaces (e.g. no half-space can reproduce the "locking" effects described above).

The additional problem with springs and dashpots arises when the integral stiffness is distributed over the contact surface $Q$. Physically in every point of $Q$ there are no distributed angular loads impacting basement from the soil. So, only translational springs and dashpots are usually distributed over $Q$, and angular impedances are the results of these distributed translational springs. For a surface basement vertical distributed springs are responsible for rocking impedances, and horizontal springs are responsible for torsional impedance. The problem here is that all attempts to find the distribution shape for vertical springs to represent rocking impedances simultaneously with vertical one have failed. If fact, integral rocking stiffness obtained from distributed vertical springs is always less than actual rocking stiffness; on the contrary, integral rocking damping obtained from distributed vertical dashpots is always greater than the actual one. Physical reason of this mismatch is the interaction between different points through soil. Spring/dashpot model is "local" in nature: the response is determined by motion of this very point, and not neighbors. This is not physically true.

The author found a way to treat both problems at once. The idea is to work in the time domain using a platform model of Fig.5 with conventional springs and dashpots (lumped or 
distributed). Of course we get some "platform" impedances $D(\omega)$, different from "wave" impedances $C(\omega)$, but the idea is to tune the platform excitation $V_{b}$ so to account for the difference between "wave" impedances and "platform" impedances. Six components of the platform excitation may be tuned to reproduce six components of response - e.g., six components of the rigid base mat's accelerations. Such an approach combines the calculations in the frequency domain (platform seismic input) with calculations in the time domain (final dynamic analysis of the platform model), that is why this method is called "combined". Besides, this method is "exact" for rigid base mats only: the stiffer is a base mat, the more accurate are the results. That is why this method is also called "asymptotic" full name is "combined asymptotic method" (CAM) [22].

The last item to discuss in this part is practical tools to obtain impedances and seismic loads (or weightless base mats' motions) in the frequency domain. At the moment the author uses one of two computer codes. For rigid surface basements on a horizontally-layered soil code CLASSI is the most appropriate. For the embedded basements with possible local breaks in horizontal layering code SASSI is used (SASSI can be used for surface base mats also, but is more sophisticated).

In both cases formula (3) is a basic equation for impedances, and the dynamic stiffness matrix Go linking set of nodes in the infinite soil is a key issue (the second matrix Gint is absent for surface basement in CLASSI and easily obtained by FEM for the embedded basement in SASSI). To get Go, they first obtain a dynamic flexibility matrix, describing displacements due to the unit forces (this is a Green's function). Here is a difference between two codes.

Professor J.Luco managed [7] to develop Green's function analytically for the case of surface load and surface response node in horizontally-layered soil in the frequency domain. Then contact surface $Q$ was covered with number of rectangular elements of different shapes. Loads were applied in the centers of each element one by one; response displacements were obtained in the centers of each element for each load. The additional convenience was that for the horizontally layered soil one can shift the loaded node and the response node horizontally, and the link between them stays the same. So, in fact one needs Green's functions only for a single loaded node and for the response nodes placed at a distance from the loaded node within maximal size of the mat. Zero (for vertical load) and first (for horizontal load) Fourier terms describe the displacement field along angular cylindrical coordinate, enabling to store Green's functions only along 1D radial line. Thus, it is not very time-consuming to obtain Green's function (it is done in a separate module "Green") and to use it in order to obtain the full flexibility matrix (in separate module "Claff"). Then this full flexibility matrix is turned into a full stiffness matrix $G_{0}$. Finally $G_{0}$ is condensed to the $6 \times 6$ impedance matrix $C$. The whole procedure is repeated for each frequency of the prescribed set. For a surface rigid basement and vertical waves there is no separate problem to find the load matrix $B$ in equation (4): kinematical interaction does not change control motion, so $B$ consists of the first three columns of $C$. This was a brief description of CLASSI ideology. 

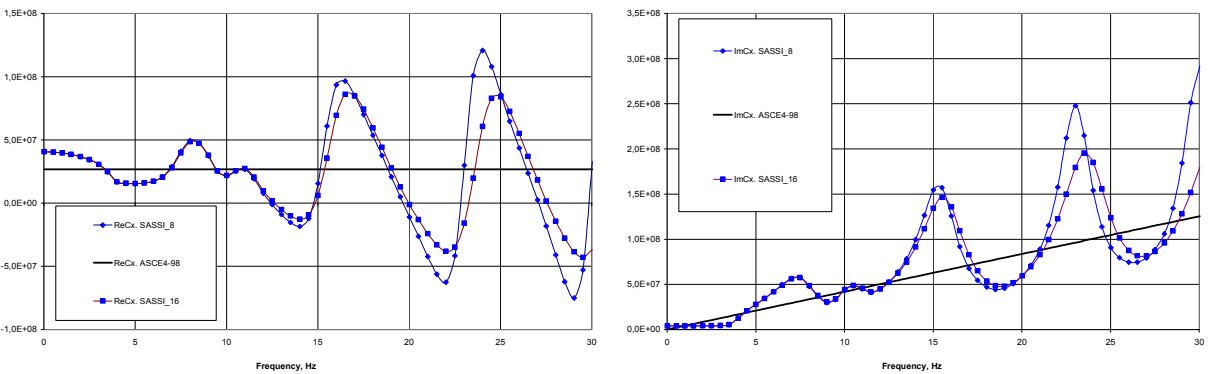

Real part of vertical translational impedances
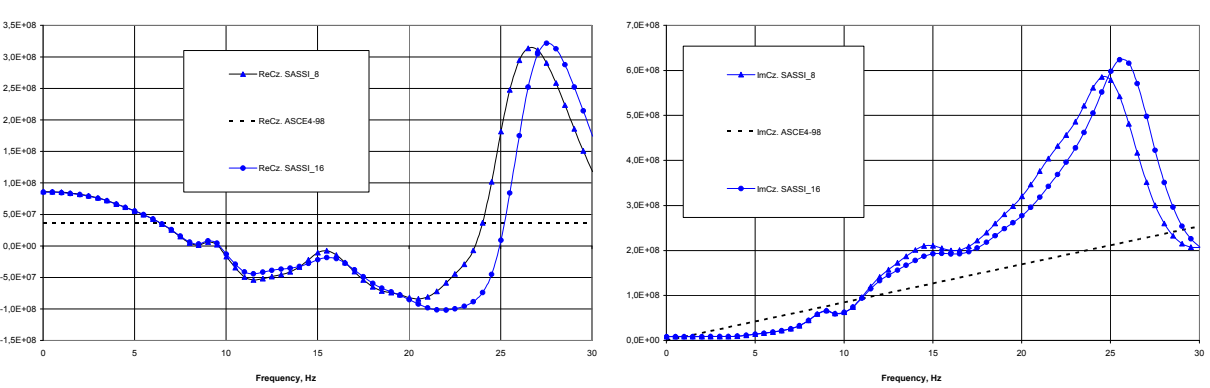

Rel part of rocking impedances

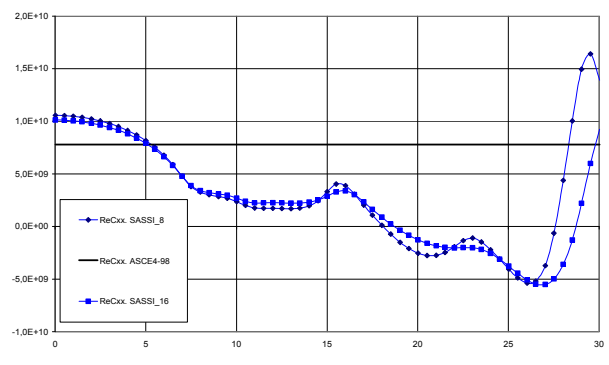

Inainary part of rocking impedances
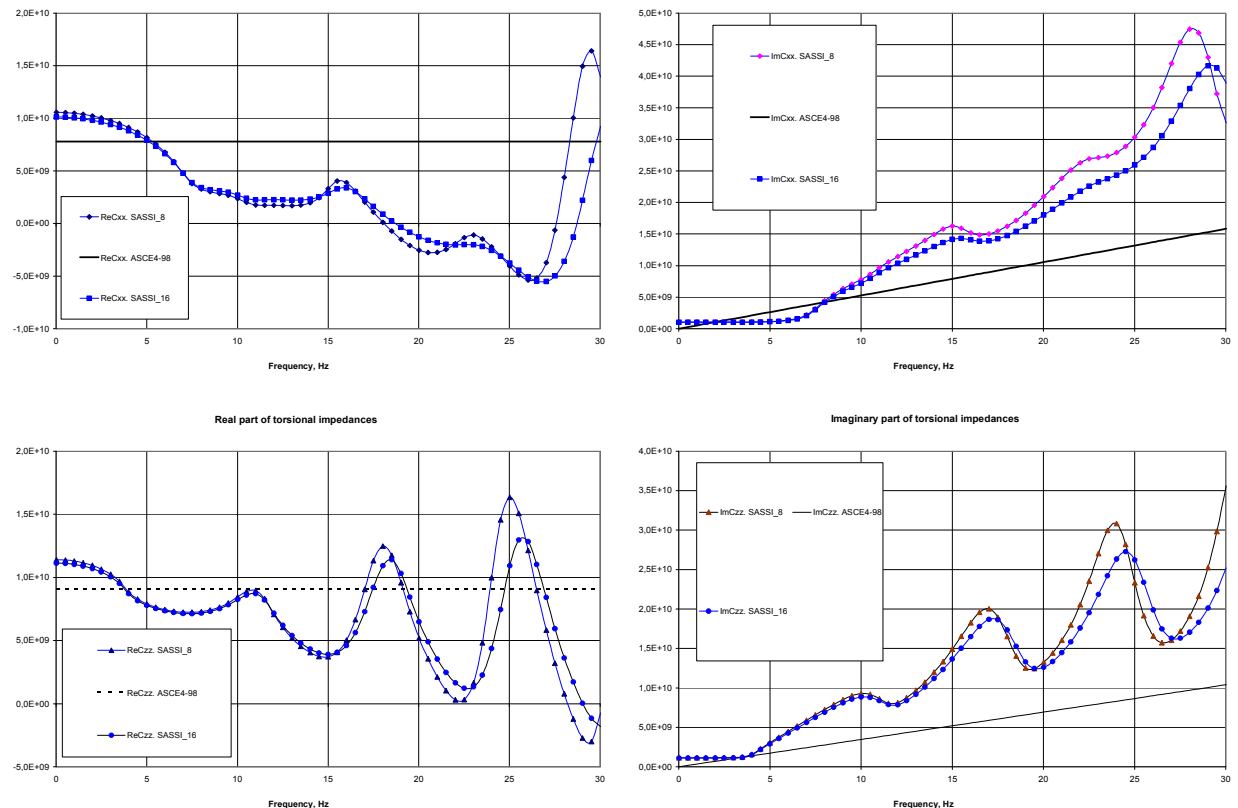

Figure 11. Impedances for a basement $30.6 \times 30.6$ meters on the surface of homogeneous layer with mass density $\mathrm{Q}=2 \mathrm{t} / \mathrm{m}^{3}$, wave velocities $\mathrm{V}_{\mathrm{s}}=400 \mathrm{~m} / \mathrm{s}, \mathrm{V}_{\mathrm{p}}=1100 \mathrm{~m} / \mathrm{s}$, internal damping $\gamma=5 \%$, thickness $\mathrm{h}=26$ $\mathrm{m}$, underlain by rigid rock 
J.Lysmer for the same problem (but for the embedded loaded and response nodes) used direct approach described above. He made $V_{\text {int }}$ a cylindrical column, with just a single element per radius. The bottom of this column was placed on a homogeneous half-space, so a dashpot was put there in each of three directions. Lateral boundaries were of Waas type. Loads were applied to the nodes at the vertical axis of cylinder. The response nodes were either at the same axis or out of the cylinder (for regular mesh the radius of cylinder was set equal to 0.9 of mesh size). In the first case, the response displacements were obtained from the FEM problem. In the second case the solution of the FEM problem gave the displacements of the nodes at the lateral boundary. Then the displacements in the whole infinite volume $V_{\text {ext }}$ were obtained using "homogeneous wave fields" in cylindrical coordinates described above (with Hankel's functions along radius). Like in CLASSI, horizontal shift of the loaded and response nodes is allowed in the horizontally-layered soil. So only a single cylinder has to be studied. Like in CLASSI, it is done in a separate module (in SASSI it is called POINT). After a flexibility matrix for a set of "interacting nodes" covering Vint is obtained, it is turned into a stiffness matrix $G_{0}$. This matrix may be condensed into a part of the impedance matrix like in CLASSI (another part comes from Gint).

However, one can instead return to the "problem A2" without condensation (e.g., for flexible basement or locally modified soil around the basement). Bottom of surface $Q$ is placed not on the rock, as it was in Fig.3, but where it is convenient (usually at the bottom of the modified soil volume, if any, or at the bottom of the basement). This approach may be called a "combined" one, because the direct approach is used to get Green's function only; further on, the impedance approach (extended for flexible underground volume $V_{\text {int }}$ ) is applied.

The problem of axial symmetry has gone both in CLASSI and in SASSI: though "basic problem" in Green or in POINT has an axisymmetric geometry and is solved in cylindrical coordinates, the "interaction nodes set" may be arbitrary in shape.

Another problem for SASSI is with flexible underlying half-space: Waas boundaries were developed for rigid half-space at the bottom only. Lysmer's team found a brilliant approximate solution. Energy for a cylinder in POINT is taken down in the half-space by vertical body waves (modeled by dashpots, see above) and by surface Rayleigh waves in the flexible half-space. These Rayleigh waves in the frequency domain have a certain depth where the wave displacements are close to zero. One can put "rigid" bottom at that very depth without spoiling the response in the upper part of the soil. The problem is that this depth is frequency-dependent: so, the model in POINT becomes non-physical. However, transmitting boundaries, as we remember, were developed by G.Waas in the frequency domain, so the problem is solved frequency by frequency. Hence, one can change the depth of "rigid boundary" at the bottom of the model according to the frequency change. In SASSI it is done automatically: soil model in POINT consists of the upper part with fixed layers, and of the lower part with frequency-dependent layers.

For surface basements one can compare the impedances given by SASSI and CLASSI. In the frequency range below $15 \mathrm{~Hz}$ the results are very close to each other. Examples were shown above (see Fig.10). 


\section{Soil non-linearity - SHAKE ideology - Contact non-linearity}

Soil in reality is considerably non-linear. That is why H.B.Seed [23] suggested special method to describe seismic response of horizontally-layered soil to the verticallypropagating free-field seismic waves by equivalent linear model. Parameters of this model are obtained in iterative calculations, as modules and damping in each layer are straindependent. This method is used in the famous SHAKE code [24]. Usually 7-8 iterations are enough to get the error less than $1 \%$.

SHAKE calculations may be started from the surface (i.e. surface motion is given; for each other layer the motion is to be calculated together with equivalent soil properties); this case is called "deconvolution". On the contrary, SHAKE calculations may be started from some depth where the motion is given; this case is called "convolution". Motion for one and the same point in depth may be given in one of two variants. The first variant is just a motion of the point inside soil composed of the upcoming wave and wave coming down (see the 1D example in p.5 above). The second variant is to set just the upcoming wave amplitude. Sometimes this motion is called "outcropped" motion, because they double the upcoming wave amplitude, like it is at the free surface (see the example in p. 6.1 above). However, such a "doubled" motion will be similar to the motion of the really outcropped surface only at the surface of the underlying homogeneous half-space, and not in the middle of the layered soil.

The typical case consists of two sequential SHAKE calculations. First, deconvolution is performed using the initial soil model and initial seismic motion of the free surface of the initial soil (the author usually calls it "foundation no.1"). Note, that the underlying halfspace is not modified in the process of SHAKE iterations. That is why one should include into the modified part at least one upper layer of half-space. After the deconvolution is through, one should change the half-space manually making parameters equal to the lowest modified layer. The main result of deconvolution is an "outcropped" motion of the halfspace surface. The second stage is a convolution. Half-space stays the same as in deconvolution (after manual modification), but the upper layers may change due to various reasons starting from the direct changes in the construction process (very often the upper several meters of the initial soil are withdrawn). Sometimes the weight of the future structure changes the properties of the soil. Anyhow, we generally have "foundation no.2" instead of the first soil foundation. In the convolution procedure the outcropped motion of the half-space surface is given, and the whole wave field with equivalent soil properties is obtained as a result together with motion of the new surface.

Typical strain-dependent characteristics of different soils are shown on Fig.12. Sometimes they call it "degradation curves".

One should remember that degradation curves for shear modulus $G$ are given in relative terms $\mathrm{G} / \mathrm{G}_{0}$, where $\mathrm{G}_{0}$ is a low-strains value. On the contrary, strain-dependent characteristics of the internal damping are given for dimensionless coefficients without using low-strain values. 

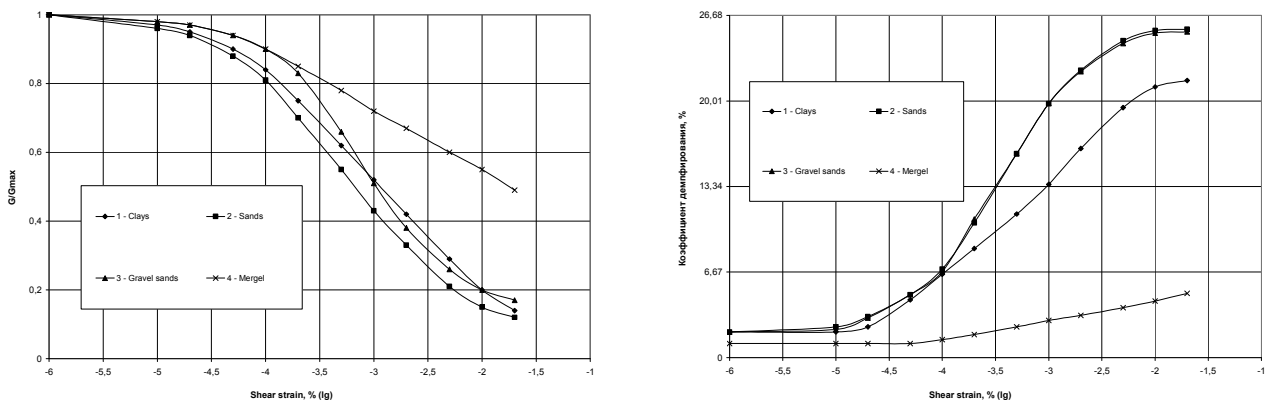

Figure 12. Typical strain-dependent characteristics of soils

Typical results of SHAKE calculations for three levels of seismic intensity are given on Fig.13. Abbreviations of the level names mean: OBE - operational basis earthquake; SSE safe shutdown earthquake (operations and shutdown refer to nuclear reactors activity). The results are calculated separately in two vertical planes (OXZ and OYZ) and then averaged (usually in two planes they are close to each other for every layer).
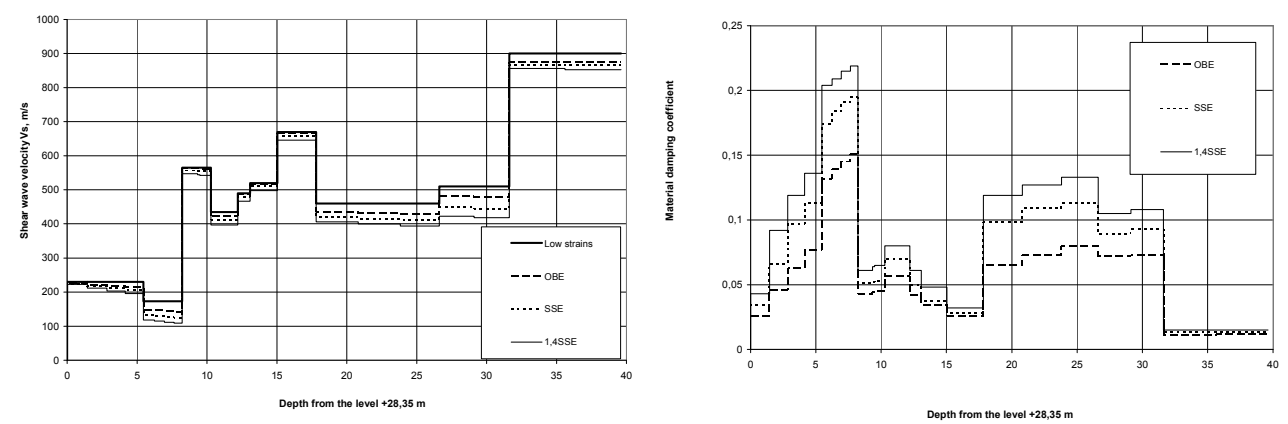

Figure 13. Typical results of the SHAKE calculations

We see that near the surface the equivalent soil characteristics are close to the low-strain values. This is because of zero strains at the free surface for every intensity level.

Usually the greatest relative shift in velocities and damping is at the bottom of soft soil layers. If the intensity is very high, the iterations during deconvolution may not converge. Physically it means that the given surface motion is no longer compatible with given soil properties; such motion cannot be transferred by given soil to the surface. 
Both SHAKE results - profiles of wave velocities/damping and surface motion - are further on used in SSI analysis.

Theoretically a wide range of nonlinearities can be described by strain-dependent properties. However, these properties should be transient, i.e. they should vary from one time point to another during one seismic event. In the Seed's approach these properties in each soil layer are established once for the whole event duration (changing not from time to time, but from one linear run to another one). So, in fact soil properties depend not on the instant transient strains, but on some "effective" strains (in practice - on some portion of the maximal strain over the duration). In fact, Seed provided a tool to extrapolate the results of the lab test with harmonic excitation of the soil sample to another situation with nonharmonic wave in the same sample.

Nowadays seismologists are ready to provide more sophisticated approaches to the soil description. They can model accelerations in the soil more accurately with truly transient soil properties. But in the SSI problems (e.g., in SASSI or CLASSI) one will need linear soil with "effective" soil properties, so SHAKE still remains the best "pre-SSI" processor.

The result of SHAKE is obtained for horizontally-layered soil without structure. So, they say, that it contains "primary" non-linearity. The same equivalent strain-dependent properties can be further applied in the SSI problems to account for the "secondary" nonlinearity caused by structure, but the result will spoil horizontally-layered geometry of the soil. As we know, that will spoil CLASSI model and create additional problems in SASSI model.

That is why modern standards (e.g., ASCE4-98) require the consideration of the primary non-linearity, but do not require consideration of the secondary non-linearity of the soil.

Looking at soil-structure system, one can find nonlinearities not only in soil, but at the contact surface. Even if both soil and structure are modeled by linear systems, they may have various contact terms. Most often full contact is assumed - it does not spoil linearity. However, soil tension (unlike soil compression) is very limited, and that may cause a) uplift of the base mat from the soil, b) separation of the embedded basement walls from the soil.

Base mat uplift may be estimated if linear "full contact" vertical forces over contact surface $\mathrm{Q}$ are compared to the static vertical forces caused by structural weight. In practice the full uplift is seldom met, but rocking of the structure can cause dynamic tension near the edges of the base mat. This "partial" dynamic uplift usually occurs for stiff soils and sizable structures. Does it change the response motion considerably? Today they believe that if the area of partial dynamic uplift is less than $1 / 3$ of the total contact area, one can neglect this uplift and still use SSI linear model with full contact.

Separation of vertical embedded walls is treated as follows. In the upper half of the embedment depth (but only up to 6 meters from the surface) they break soil-structure contact completely. Below this level they assume full contact. 


\section{Non-mandatory assumptions}

So, basic assumptions currently used in the SSI analysis are a) linearity of the soil, of the structure and of the soil-structure contact; b) horizontal layering of the soil (except some limited volume near the structure).

There are two other assumptions - not mandatory, but usually used in the SSI analysis. The first assumption is the rigidity of the soil-structure contact surface. Usually base mats are not extremely rigid, but they are considerably enforced by rather dense and thick shear walls, so in fact their behavior is almost rigid. Standards ASCE4-98 allow the treatment of base mats of the NPP structures as rigid ones. However, SASSI can treat flexible base mats as well. Different parameters of structural seismic response show different sensitivity to the flexibility of the base mat. Some examples are presented in the author's reports in SMiRT-21 [25,26].

The second assumption is about seismic wave field in the soil without structure. Usually, one starts from the three-component acceleration recorded on the surface of the soil (in some "control point", as they say). As we saw, in SSI problem one needs to know the motion of a certain soil volume (at least the soil motion in the nodes of the future contact surface). So some additional assumptions are introduced. The most common assumption "is vertically propagating body seismic waves in horizontally-layered medium". This assumption means, that three components of the acceleration in the control point are produced by three separate vertically propagating waves: vertical acceleration is a result of the P-wave, two horizontal accelerations are the result of two S-waves in main coordinate vertical planes. Each wave can be analyzed separately by SHAKE, providing seismic motion of any point in depth. Another consequence of this assumption is that seismic motion depends on vertical coordinate, but not on the horizontal coordinates: every horizontal plane in the free field moves "rigidly".

Again, this assumption is not mandatory. In SASSI one can set up other assumptions linking the whole wave field to the control point motion. However, as SHAKE (usually used as a preprocessor for SASSI) implements that very assumption, most often this assumption is accepted for the whole SSI problem.

\section{Some examples of the SSI effects in practice}

Concluding the chapter, the author should like to give some practical examples. One of recently built NPPs was analyzed for different soil and excitation models. Let us look at the acceleration response spectra on the elevation $+21.5 \mathrm{~m}$.

The first comparison is for rigid soil and flexible soil (without embedment). As we remember, rigid soil means the absence of the SSI effects. Flexible soil in this case was of medium type. Structure was one of the NPP buildings. One and the same three-component seismic excitation (corresponding to the standard spectra described in RG1.60) was applied 
to the surface of the rigid and flexible soil. In the first case this very motion became the response motion of the base mat; in the second case, the response motion of the base mat was modified by the SSI effects. The comparison of the floor response spectra (enveloped over 8 corner nodes of the floor slab, smoothed and broadened $15 \%$ each side in the frequency range) at the structural level $+21.5 \mathrm{~m}$ is shown in Fig. 14.

We see the considerable difference in the spectral shape: SSI effects form the main spectral peak, but for high frequency range (here - after $5 \mathrm{~Hz}$ ) spectral accelerations with SSI are less than without SSI. As to the maximal accelerations (we see them in the right part of the spectral curves), SSI effects may decrease them (see Fig.14b) or not. It sometimes depends on the height of the floor considered.
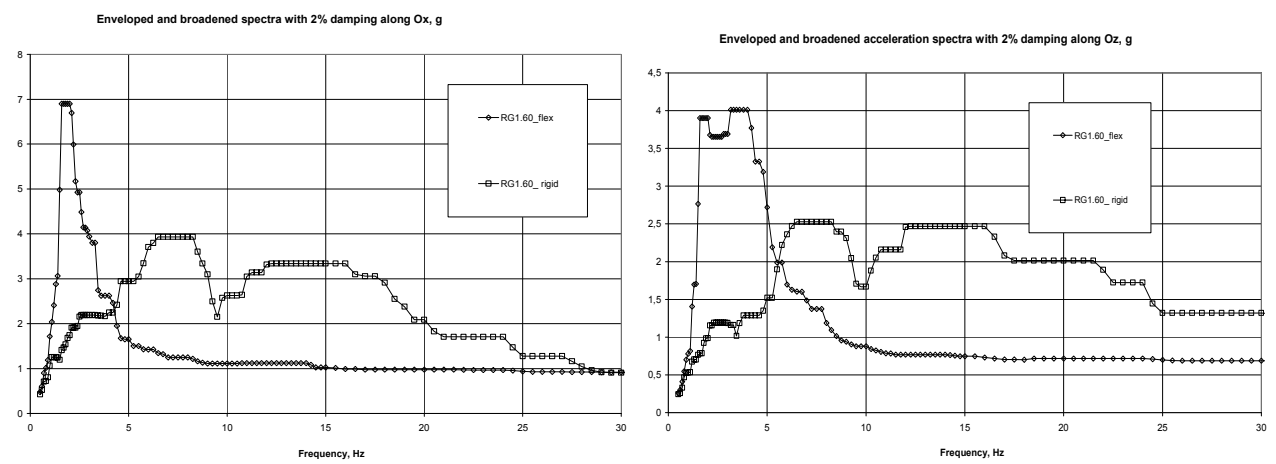

Figure 14. Comparison of enveloped broadened spectra with $2 \%$ damping at the level $+21.5 \mathrm{~m}$ for excitation RG1.60 for rigid and flexible soil; spectral accelerations are shown along horizontal axis Ox (a) and vertical axis $\mathrm{Oz}(\mathrm{b})$

The second comparison is for surface and embedded base mats. Site-specific threecomponent seismic excitation is "applied" at the free surface of two flexible soil foundations: first, real soil foundation; second, the same soil foundation without upper 10.4 meters of soil (corresponding to the embedment depth of the structure). The comparison of the response spectra is shown in Fig.15 in the same format as in Fig.14.

We see that the embedment considerably impacts the first spectral peaks, decreasing spectral accelerations. The physical reason is that the mass of the "outcropped soil" for the embedded basement in fact is subtracted from the mass of the basement when inertial loads are developed for the platform model of "inertial interaction" in the moving coordinate system placed on the platform. 

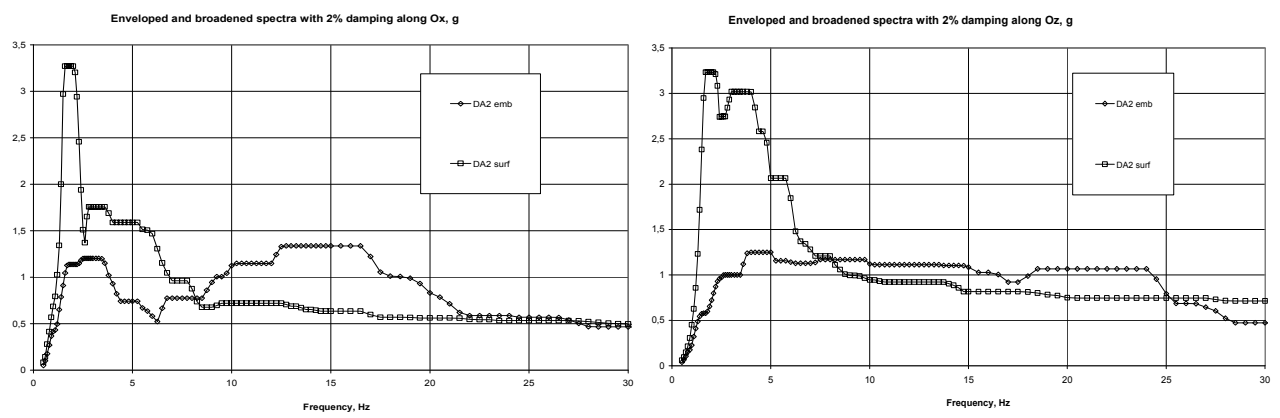

Figure 15. Comparison of enveloped broadened spectra with $2 \%$ damping at the level $+21.5 \mathrm{~m}$ for sitespecific excitation for surface and embedded structures; spectral accelerations are shown along horizontal axis $\mathrm{Ox}(\mathrm{a})$ and vertical axis $\mathrm{Oz}(\mathrm{b})$

On the other hand, for higher frequencies there may appear spectral accelerations for the embedded structure greater than those for the surface structure (see Fig.14a). Still the overall effect of the small embedment is considered conservative; that is why standards ASCE4-98 [1] allow neglecting the embedment, if the embedment depth is less than $30 \%$ of the equivalent radius of the basement. Interesting to note that in the sample shown in Fig.15, this limit is almost met (10.4 m of embedment versus $34.7 \mathrm{~m}$ of equivalent radius). Nevertheless, the difference in the spectral shape in Fig. 15 is dramatic.

\section{Conclusions}

Concluding the chapter about soil-structure interaction (SSI), the author should like to give several recommendations to engineers.

1. At the very beginning one should estimate the importance of SSI and decide whether it should be considered at all. The answer depends on the soil data (wave velocities in the soil, first of all), base mat size/embedment and inertia of the structure. For civil structures most often SSI can be omitted (i.e. structure can be analyzed using a platform model with platform kinematical excitation given by seismologists and without soil springs).

2. If SSI is to be considered, one should examine whether some simple assumptions can be applied. Main assumptions: homogeneous half-space or a layer underlain by rigid rock as a soil model (depends on real-world geotechnical data), surface base mat, rigid base mat. General recommendation is as follows. One should start with the simplest model allowed by standards. Only if the results seem overconservative, one should try to go to more sophisticated models, accounting to various specific SSI effects. Usually there is a trade between the complexity of the model and the conservatism of the results. 
3. SSI effects are frequency-dependent. Most of effects are valid in a certain frequency range. Out of this range they may lead to the opposite changes.

4. SSI analysis requires special tools. General-purpose codes, including FEM soft, cannot treat SSI properly because of the infinite geometry of the initial problem. Specialpurpose tools like CLASSI, SASSI, etc. should be used.

5. If direct approach is used, special attention should be paid to the boundaries. Preliminary analysis of test examples (e.g., initial soil without structure with the same boundaries and excitation) is strongly recommended.

6. Wave nature of SSI effects requires special attention when FEM is used: element size for the soil and time step must be compared with frequency ranges of interest. Otherwise, the most significant effects may be missed.

7. Non-linearity of different kinds is to be treated properly. Primary non-linearity of the soil is handled by SHAKE. Contact non-linearity is treated approximately as described above. If a structure itself is considerably non-linear, usually one has to omit wave SSI at all.

Nowadays, the research goes forward. The current goal is to combine non-linearity inside $\mathrm{V}_{\text {int }}$ (including contact non-linearity and structural nonlinearity) with linearity of infinite soil in $V_{\text {ext. }}$

\section{Author details}

Alexander Tyapin

"Atomenergoproject", Moscow, Russia

\section{References}

[1] Seismic Analysis of Safety-Related Nuclear Structures and Commentary. ASCE4-98. Reston, Virginia, USA. 1999.

[2] A Methodology for Assessment of Nuclear Power Plant Seismic Margin (Revision 1). EPRI NP-6041-SL. Revision 1. Project 2722-23. August 1991. California, USA.

[3] Reissner E (1936) Stationare, axialsymmetriche durch eine Shuttelnde Masse erregte Schwingungen eines homogenen elastischen Halbraumes. Ingenieur-Archiv. 7/6: 381396.

[4] Luco J.E (1982) Linear Soil-Structure Interaction: A Review. Earthquake Ground Motions and Its Effects on Structures. Applied Mechanics Division, ASME 53: 41-57.

[5] Gulkan P, Clough R /Editors (1993) Developments in dynamic soil-structure interaction/Ed. NATO Advanced Institutes Series. Series C: Mathematical and Physical Sciences. Vol.390. Dordrecht/Boston/London: Kluwer Academic Publishers. 439 p.

[6] Seed H, Lysmer J (1977) Soil-Structure Interaction Analysis by Finite Element Method. State of the Art. Transactions of the International Conference on Structural Mechanics in Reactor Technology (SMiRT-4). San Francisco. Vol.K. K2/1. 
[7] Luco J (1976) Vibrations of a Rigid Disc on a Layered Viscoelastic Medium. Nuclear Engineering and Design. 36: 325-340.

[8] Kausel E, Roesset J, Waas G (1975) Dynamic Analysis of Footings on Layered Media. J. of Engineering Mechanics Div., ASCE. 101. EM5: 679-693.

[9] Lysmer J, Tabatabaie R, Tajirian F, Vahdani S, Ostadan F (1981) SASSI - A System for Analysis of Soil-Structure Interaction. Research Report GT 81-02. University of California, Berkeley.

[10] Wolf J.P (1985) Dynamic Soil-Structure Interaction. Prentice-Hall, Englewood Cliffs, NJ.

[11] Wolf J.P (1988) Soil-Structure Interaction Analysis in Time Domain. Prentice-Hall, Englewood Cliffs, NJ.

[12] Kausel E, Roesset J (1977) Semianalytic Hyperelement for Layered Strata. J. of Engineering Mechanics Div., ASCE. 103. EM4: 569-588.

[13] Kuhlemeyer R, Lysmer J (1973) Finite Element Method Accuracy for Wave Propagation Problems. J. of Soil Mechanics and Foundations Div., ASCE. 99. SM5: 421-427.

[14] Lysmer J, Kuhlemeyer R (1969) Finite Dynamic Model for Infinite Media. J. of Engineering Mechanics Div., ASCE. 95. EM4: 859-877.

[15] Lysmer J, Udaka T, Seed H, Hwang R (1974) LUSH - a Computer Program for Complex Response Analysis of Soil-Structure Systems Rep. EERC 74-4. Berkeley, California.

[16] Lysmer J, Waas G (1972) Shear Waves in Plane Infinite Structures. J. of Engineering Mechanics Div., ASCE. 98. EM1: 85-105.

[17] Waas G (1972) Linear Two-Dimensional Analysis of Soil Dynamics Problems in Semiinfinite Layered Media. Ph.D. Dissertation. University of California, Berkeley.

[18] Lysmer J, Udaka T, Tsai C, Seed H (1975) FLUSH - A Computer Program for Approximate 3-D Analysis of Soil-Structure Interaction Problems. Report No.75-30. University of California, Berkeley.

[19] Berger E, Lysmer J, Seed H (1975) ALUSH - A Computer Program for Seismic Response Analysis of Axisymmetrical Soil-Structure Systems. Report No.75-31. University of California, Berkeley.

[20] Tyapin A (2007) The frequency-dependent elements in the code SASSI: a bridge between civil engineers and the soil-structure interaction specialists. Nuclear Engineering and Design. 237: 1300-1306.

[21] Tyapin A (2011) Modal approach to the condensed dynamic stiffness evaluation // Journal of Mechanics Engineering and Automation. 1. No.1: 1-9.

[22] Tyapin A (2010) Combined Asymptotic Method for Soil-Structure Interaction Analysis. J. of Disaster Research. 5. No.4: 340-350.

[23] Schnabel P, Seed H, Lysmer J (1972) Modifications of Seismograph Records for Effects of Local Soil Conditions. Bulletin of Seismological Society of America. 62. N 6: 16491664.

[24] Schnabel P, Lysmer J, Seed H (1972) SHAKE - a Computer Program for Earthquake Response Analysis of Horizontally Layered Sites. Rep. EERC 72-12. Berkeley, California. 
[25] Tyapin A (2011) The effects of the base mat's flexibility on the structure's seismic response. Part I: wave solution. SMiRT21. New Delhi. \#85.

[26] Tyapin A (2011) The effects of the base mat's flexibility on the structure's seismic response. Part II: platform solutions. SMiRT21. New Delhi. \#266. 